\title{
X-ray obscuration and obscured AGN in the local universe ${ }^{\star}$
}

\author{
M. Guainazzi ${ }^{1}$, G. Matt $^{2}$, and G. C. Perola ${ }^{2}$ \\ 1 XMM-Newton Science Operations Center, European Space Astronomy Center, ESA, Apartado 50727, 28080 Madrid, Spain \\ e-mail: mguainaz@xmm.vilspa.esa.es \\ 2 Università degli Studi “Roma Tre”, Via della Vasca Navale 84, 00146, Roma, Italy
}

Received 16 June 2005 / Accepted 4 August 2005

\section{ABSTRACT}

We discuss the X-ray properties of 49 local $(z<0.035)$ Seyfert 2 galaxies with HST/WFC2 high-resolution optical coverage. It includes the results of 26 still unpublished Chandra and XMM-Newton observations, which yield 25 (22) new X-ray detections in the $0.5-2 \mathrm{keV}$ (2-10 keV) energy band. Our sample covers a range in the $2-10 \mathrm{keV}$ observed flux, $F_{2-10}$, from $3 \times 10^{-11}$ to $6 \times 10^{-15} \mathrm{erg} \mathrm{cm}^{-2} \mathrm{~s}^{-1}$. The percentage of objects that are likely obscured by Compton-thick matter (column density, $N_{\mathrm{H}} \geq \sigma_{t}^{-1} \simeq 1.6 \times 10^{24} \mathrm{~cm}^{-2}$ ) is $\simeq 50 \%$, and reaches $\simeq 80 \%$ for $\log \left(F_{2-10}\right)<12.3$. Hence, $\mathrm{K}_{\alpha}$ fluorescent iron lines with large Equivalent Width $(E W>0.6 \mathrm{keV})$ are common in our sample (6 new detections at a confidence level $\geq 2 \sigma$ ). They are explained as due to reflection off the illuminated side of optically thick material. We confirm a correlation between the presence of a 100-pc scale nuclear dust in the WFC2 images and Compton-thin obscuration. We interpret this correlation as due to the large covering fraction of gas associated with the dust lanes. The X-ray spectra of highly obscured AGN invariably present a prominent soft excess emission above the extrapolation of the hard X-ray component. This soft component can account for a very large fraction of the overall X-ray energy budget. As this component is generally unobscured - and therefore likely produced in extended gas structures - it may lead to a severe underestimation of the nuclear obscuration in $z \sim 1$ absorbed AGN, if standard X-ray colors are used to classify them. As a by-product of our study, we report the discovery of a soft X-ray, luminous $\left(\simeq 7 \times 10^{40} \mathrm{erg} \mathrm{s}^{-1}\right)$ halo embedding the interacting galaxy pair Mkn 266

Key words. galaxies: active - galaxies: nucleus - galaxies: Seyfert - X-ray: galaxies

\section{Introduction}

While it is widely accepted that "type 2" Active Galactic Nuclei (AGN) are generally obscured in X-rays by high column densities, $N_{\mathrm{H}}$, the exact location and distribution of the obscuring gas are still debated. The obscuring gas is identified with matter responsible for orientation-dependent effects (e.g., the dusty compact molecular "torus" of the AGN Unification Scenarios; Antonucci \& Miller 1985; Antonucci 1993). However, recent observational results challenge this interpretation. Fast changes in the X-ray column density advocate rather for matter closer to the nuclear supermassive black hole, such as the Broad Line Region (BLR; Elvis et al. 2004; Lamer et al. 2003). "Narrow" components of the $\mathrm{K}_{\alpha}$ fluorescent iron lines in unobscured AGN - when resolved by high-resolution spectroscopic Chandra measurements - are sometimes consistent with an origin in the BLR as well (Yaqoob et al. 2001, 2004; Bianchi et al. $2003 \mathrm{~b}$ ), providing evidence for the existence of obscuring matter on that scale. However, both the low covering fraction of the BLR and their optical thickness seem too low to account for the large fraction of heavily X-ray obscured AGN (e.g. Risaliti et al. 1999). Other pieces of evidence - again mainly based

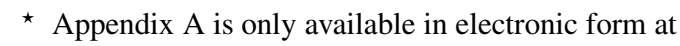
http://www. edpsciences.org on X-ray variability studies - suggest a complex structure for the X-ray obscuring matter in many instances, and in particular rules out a single homogeneous, azimuthally-symmetric obscuring structure (Matt et al. 2003a; Guainazzi et al. 2005a). Although an inhomogeneous "patchy" torus (Pier \& Krolik 1992) is still consistent with the data, and with the presence of Compton-thick reflection in unobscured AGN (Bianchi et al. 2004), a possible scenario is that X-ray obscuration occurs on various scales, sometimes due to more compact matter close to the black hole, sometimes due to matter associated with the host galaxy rather then with the nuclear environment. In a few cases, "multiple absorbers" are directly measured in X-rays (Matt et al. 2001, 2003a), and their lowest column densities are consistent with the optical reddening due to host galaxy dust lanes, once a standard Galactic gas-to-dust ratio is assumed ${ }^{1}$.

Another possible approach to elucidate the location and geometrical distribution of X-ray obscuring matter in AGN is a comparison between the distribution of X-ray column densities and indicators of reddening and/or extinction at other wavelengths. Prior to the launch of the major X-ray observatories

\footnotetext{
1 On a possible systematic difference in the gas-to-dust ratio between our Galaxy and luminous nearby AGN, see Granato et al. (1997, and references therein).
} 
Chandra and XMM-Newton this task was hampered by the limited number of objects for which X-ray spectroscopic measurements were available.

Guainazzi et al. (2001, GO1 hereafter) studied the correlation between X-ray obscuration and the presence of nuclear dust in the innermost $\sim 100 \mathrm{pc}$ around the nucleus, as detected in the high-resolution WFC2/HST imaging survey of nearby Seyfert galaxies after Malkan et al. (1998, M 98 hereafter). G01 discovered a possible correlation: Compton-thin objects tend to be preferentially located in galaxies with a larger dust content, whereas the likelihood that a galaxy hosts a Compton-thick nucleus is not affected by its nuclear dust content. However, the statistical significance of the correlation in G01 was at the $1-\sigma$ level. Since then, we have carried out an XMM-Newton observing program to verify this tentative correlation on a larger sample. The results of this program, coupled with the analysis of still unpublished archival data of objects belonging to the M98 sample, are the main subject of this paper.

The structure of the paper is as follows: in Sect. 2 we introduce the sample; in Sect. 3 we present the reduction/analysis procedure. The spectral results are described in Sect. 4, and discussed in Sect. 5. Our main conclusions are summarized in Sect. 6.

In this paper: the acceptance level for a spectral fit corresponds to a null hypothesis probability $\leq 5 \%$; errors on the spectral fit parameters and derived quantities are at the $90 \%$ confidence level for 1 interesting parameter; all other uncertainties are at the 1- $\sigma$ level; energies refer to the source rest frame, unless otherwise specified.

\section{The sample}

The sample is made up of all the Seyfert 2 galaxies in the Veron catalogue (Veron-Cetty \& Veron 2003), which: a) have $z \leq 0.035$; b) have been observed in X-rays; c) have been included in the high-resolution HST/WFC2 survey of M98. The plate scale of WFC2 images (0.046) corresponds to a pixel size $\$ 32 \mathrm{pc}$, and the total image size $\left(37^{\prime \prime} \times 37^{\prime \prime}\right)$ is $\$ 25 \mathrm{kpc}$. The total number of objects fulfilling all three criteria is $49^{2}$, which represent $\simeq 16 \%$ of the total parent sample. 20 of them were discussed by G01. Although updated measurements for the GO1 sample have been used, whenever published in the literature, we did not systematically reanalyze all GO1 sources, as they are mostly well studied, X-ray bright AGN. Their X-ray absorption classification - originally derived from Bassani et al. (1999) - is therefore robust. The X-ray spectra of three more M98 objects have been published elsewhere: ESO 509-IG066 (Guainazzi et al. 2005b), NGC 424 (Matt et al. 2003a), NGC 7130 (Levenson et al. 2005). We present in Sect. 4 the results of Chandra/XMM-Newton observations of the 26 still unpublished objects belonging to the sample.

Following the classification in M98 we consider "nuclear dusty" those galaxies whose nuclear dust morphology falls into

\footnotetext{
${ }^{2}$ We have excluded from the sample UGC 4203 (the "Phoenix Galaxy"), as it belongs to the class of "Changing-look" Seyfert 2 galaxies (Matt et al. 2003b), and its classification with respect to the $\mathrm{X}$-ray obscuration is therefore ambiguous.
}

the "DC" ("Dust passing close or crossing the Center") and "DI" ("Dust Irregular") 3 categories. We have verified that the adopted classification is consistent with that reported by other authors, who discuss the same nuclear images, or images with comparable spatial resolution (Ferruit et al. 2000; Quillen et al. 1999b; Martini et al. 2003). The mean redshifts for "dusty" and "non-dusty" objects are consistent: $\langle z\rangle=0.016 \pm 0.009$ for the former, $\langle z\rangle=0.017 \pm 0.008$ for the latter. The overwhelming majority of the galaxies in our sample (86\%) are spirals. The remaining are almost equally shared between ellipticals $(5 \%)$ and irregulars (9\%).

The list of objects for which new X-ray data are presented in this paper is given in Table 1, with redshifts, $\mathrm{H}_{\alpha} / \mathrm{H}_{\beta}$ flux ratios, $\mathrm{O}[\mathrm{III}]$ fluxes and some information on the Chandra/XMM-Newton observation.

\section{Observations and data reduction}

The XMM-Newton data were reduced with SAS v6.1 (Gabriel et al. 2003), using the most updated calibration files available. In this paper, only data from the EPIC cameras (MOS; Turner et al. 2001; pn, Strüder et al. 2001) will be discussed. Event lists from the two MOS cameras were merged before accumulation of any scientific products. Single to double (quadruple) events were used to accumulate pn (MOS) spectra. High-background particle flares were removed by applying fixed thresholds on the single-event, $E>10 \mathrm{keV}, \Delta t=10 \mathrm{~s}$ light curves. These thresholds, as well as the radius of the source circular extraction regions, were optimized to maximize the signal-to-noise ratio, and are listed in Table 1 . The background was extracted from annuli around the source for the MOS, and from circular regions in the same chip for the pn, at the same height in detector coordinates as the source location. Spectra were binned in order to oversample the intrinsic instrumental energy resolution by a factor $\sim 3$. If the resulting spectral bins had less than 25 background-subtracted counts, the spectra were further rebinned to reach this limit, which ensures the applicability of the $\chi^{2}$ statistics to evaluate the quality of the spectral fitting. pn (MOS) spectra were fitted in the $0.35-15 \mathrm{keV}(0.5-10 \mathrm{keV})$ spectral range.

The Chandra data were reduced with CIAO 3.1, starting from the linearized "level 2" event lists, using CALDB v.2.28. The ACIS-S spectra were rebinned according to the same criteria employed for the EPIC ones. Fits were performed in the $0.1-11 \mathrm{keV}$ energy band.

Out of the 26 objects in Table 1, 25 (22) were detected at a level higher then $3 \sigma$ in the $0.5-2 \mathrm{keV}(2-10 \mathrm{keV})$ energy band.

\section{Spectral results}

Previous studies show that X-ray spectra of obscured AGN are complex (Turner et al. 1997; Risaliti 2002; Guainazzi et al. 2005a). This holds for the objects discussed in this paper as well. In Figs. 1 and 2 we show the sample source spectra and their residuals when a photoelectrically absorbed powerlaw model is applied. This simple model leaves significant

\footnotetext{
${ }^{3}$ I.e., "not associated with any spiral arm pattern", M 98.
} 
Table 1. Properties of the Seyfert 2 galaxies discussed in this paper. The "Date" indicates the observation date. $T_{\text {exp }}$ is the net exposure time in the pn (ACIS) XMM-Newton (Chandra) camera. The "Threshold" was applied on the XMM-Newton EPIC E > $10 \mathrm{keV}$, field-of-view, single-event light curves employed to remove intervals of high particle background. "Radius" refers to the size of the source extraction region in MOS/pn (XMM-Newton observations) and ACIS-S (Chandra observations), respectively.

\begin{tabular}{|c|c|c|c|c|c|c|c|c|c|}
\hline Source & $z$ & $\begin{array}{c}N_{\mathrm{H}, \mathrm{Gal}} \\
\left(10^{20} \mathrm{~cm}^{-2}\right)\end{array}$ & $\mathrm{H}_{\alpha} / \mathrm{H}_{\beta}$ & $F_{\mathrm{O}[\mathrm{III}]^{a}}$ & Ref. $^{b}$ & $\begin{array}{c}\text { Date } \\
\text { (DD-MM-YY) }\end{array}$ & $\begin{array}{l}T_{\exp } \\
(\mathrm{ks}) \\
\end{array}$ & $\begin{array}{c}\text { Threshold } \\
\left(\mathrm{s}^{-1}\right)\end{array}$ & $\begin{array}{c}\text { Radius } \\
\text { (arcsecs) }\end{array}$ \\
\hline \multicolumn{10}{|l|}{ Seyfert 2 exhibiting nuclear dust } \\
\hline Mkn 266 & 0.0281 & 1.7 & 5.89 & 0.32 & 8 & $02-11-01$ & 19.7 & $\ldots$ & 2.0 \\
\hline Mkn 612 & 0.0203 & 5.0 & 6.61 & 1.80 & 8 & 19-01-04 & 9.1 & $0.35 / 1.0$ & $40 / 25$ \\
\hline NGC 4156 & 0.0225 & 2.0 & 33.33 & $\ldots$ & 5 & $22-12-00$ & 51.2 & $\ldots / \ldots$ & $40 / 40$ \\
\hline NGC 4968 & 0.0099 & 9.2 & 14.9 & 1.00 & 8 & $05-01-01$ & 4.3 & $\ldots / \ldots$ & $40 / 40$ \\
\hline NGC 7212 & 0.0266 & 5.5 & 5.01 & 7.08 & 6 & 20-05-04 & 10.4 & $\ldots / 1.0$ & $30 / 60$ \\
\hline NGC 7465 & 0.0066 & 6.0 & 5.34 & $\ldots$ & 1 & $29-07-03$ & 5.0 & $\ldots$ & 6.0 \\
\hline UGC 2456 & 0.0120 & 12.1 & 8.51 & 2.40 & 8 & $20-02-05$ & 11.7 & $0.35 / 2.0$ & $35 / 35$ \\
\hline UGC 4229 & 0.0232 & 5.2 & 18.20 & 0.40 & 6 & $05-05-03$ & 4.8 & $0.35 / 1.0$ & $20 / 40$ \\
\hline UGC 6527 & 0.0274 & 1.1 & 6.57 & 5.40 & 6 & 04-11-04 & 7.8 & $0.35 / 1.0$ & $50 / 40$ \\
\hline UGC 987 & 0.0155 & 5.7 & 5.38 & 0.30 & 2 & 23-01-04 & 14.6 & $0.35 / 1.0$ & $50 / 40$ \\
\hline \multicolumn{10}{|c|}{ Seyfert 2 not exhibiting nuclear dust } \\
\hline ESO269-G012 & 0.0165 & 13.8 & $\ldots$ & 0.50 & 7 & 06-01-04 & 17.6 & $\ldots$ & 6.8 \\
\hline 2MASS J01500266-0725482 & 0.0177 & 2.3 & 7.62 & 0.53 & 4 & 21-01-04 & 9.0 & $0.35 / 1.0$ & $45 / 45$ \\
\hline IC 3639 & 0.0109 & 5.1 & 6.14 & 3.14 & 4 & 07-03-04 & 8.7 & $\ldots$ & 5.2 \\
\hline IC 4995 & 0.0161 & 4.2 & 5.37 & 2.81 & 8 & $25-09-04$ & 6.7 & $0.35 / 1.0$ & $20 / 30$ \\
\hline IRAS $15480-0344$ & 0.0303 & 9.4 & 10.19 & 1.38 & 4 & $31-07-03$ & 2.5 & $0.35 / 2.0$ & $20 / 25$ \\
\hline NGC 1410 & 0.0253 & 8.2 & 15.0 & 0.36 & 8 & 22-01-04 & 7.8 & $0.35 / 2.0$ & $25 / 40$ \\
\hline Mkn 1 & 0.0154 & 5.5 & 3.32 & 3.47 & 4 & 09-01-04 & 9.1 & $\ldots / 2.0$ & $45 / 30$ \\
\hline NGC 17 & 0.0198 & 2.6 & 43.75 & 0.11 & 8 & $22-12-02$ & 10.6 & $0.35 / 1.0$ & $40 / 40$ \\
\hline UGC 1214 & 0.0172 & 3.0 & 4.17 & 15.85 & 8 & $15-01-04$ & 9.1 & $\ldots / \ldots$ & $32 / 32$ \\
\hline UGC 2608 & 0.0233 & 14.1 & 6.31 & 2.19 & 6 & $28-01-02$ & 1.1 & $1.0 / 2.0$ & $25 / 25$ \\
\hline NGC 5283 & 0.0104 & 1.8 & 3.80 & 2.69 & 8 & $24-11-03$ & 8.9 & $\ldots$ & 2.9 \\
\hline NGC 5427 & 0.0087 & 2.2 & $\ldots$ & $\ldots$ & $\ldots$ & $26-03-04$ & 8.8 & $\ldots$ & $\ldots^{c}$ \\
\hline NGC 5953 & 0.0066 & 3.3 & 22.0 & 8.60 & 5,9 & $29-12-02$ & 9.9 & $\ldots$ & 2.5 \\
\hline UM6 25 & 0.0250 & 4.0 & 9.17 & 2.10 & 3 & 02-07-04 & 7.4 & $\ldots / 1.0$ & $30 / 30$ \\
\hline NGC 3982 & 0.0037 & 1.2 & 4.00 & 1.51 & 8 & 03-01-04 & 9.2 & $\ldots$ & 3.7 \\
\hline NGC 591 & 0.0152 & 4.8 & 6.02 & 2.30 & 6 & $12-01-04$ & 8.8 & $0.35 / 1.0$ & $25 / 40$ \\
\hline
\end{tabular}

${ }^{a}$ In units of $10^{-13} \mathrm{erg} \mathrm{cm}^{-2} \mathrm{~s}^{-1}$.

$b$ 1.- Osterbrock et al. (1987); 2.- Dahari et al. (1988); 3.- Terlevich et al. (1991); 4.- de Grijp et al. (1992); 5.- Kennicutt et al. (1992); 6.Mulchaey et al. (1994); 7.- Mulchaey et al. (1996); 8.- Polletta et al. (1996); 9.- Risaliti et al. (1999).

${ }^{c}$ Not detected.

residuals in almost all objects. As already discussed by, e.g., Guainazzi et al. (2005a), at least two continuum components in the $0.5-10 \mathrm{keV}$ energy band are required. Fluorescent emission lines from iron and other elements, often covering a wide range of ionization stages, are often present as well, as witnessed by local excesses in the residuals of Figs. 1 and 2 at $\simeq 0.6,0.9$ and $6.4 \mathrm{keV}$.

Hence, we have defined a set of physically-motivated "baseline" models to fit the data. For Compton-thin Seyfert 2s the baseline model is expressed by the following general formula:

$F_{\text {thin }}(E)=M+f_{\mathrm{s}} A E^{-\Gamma}+\mathrm{e}^{-\sigma(E) N_{\mathrm{H}}} A E^{-\Gamma}+\sum_{i} G_{i}(E)$ where $\sigma(E)$ is the photoelectric cross-section, with solar abundances according to Andres \& Grevesse (1989); $A$ is a normalization factor; $f_{\mathrm{s}}$ (the "scattering fraction") is constrained: $0 \leq f_{\mathrm{s}} \leq 1 ; M$ is the thermal emission from a collisionally ionized plasma with temperature $T$ (we used the mekal implementation in XSPEC; Mewe et al. 1985); $G_{i}(E)$ are Gaussian profiles, corresponding to fluorescence emission lines of high$Z$ elements from $\mathrm{O}$ to $\mathrm{Fe}$, the last being by far the most important and common with its $6.4 \mathrm{keV}$ neutral fluorescent line. For "Compton-thick" objects, the baseline model is slightly different:

$F_{\text {thick }}(E)=M+B E^{-\Gamma}+R(E, \Gamma)+\sum_{i} G_{i}(E)$ 

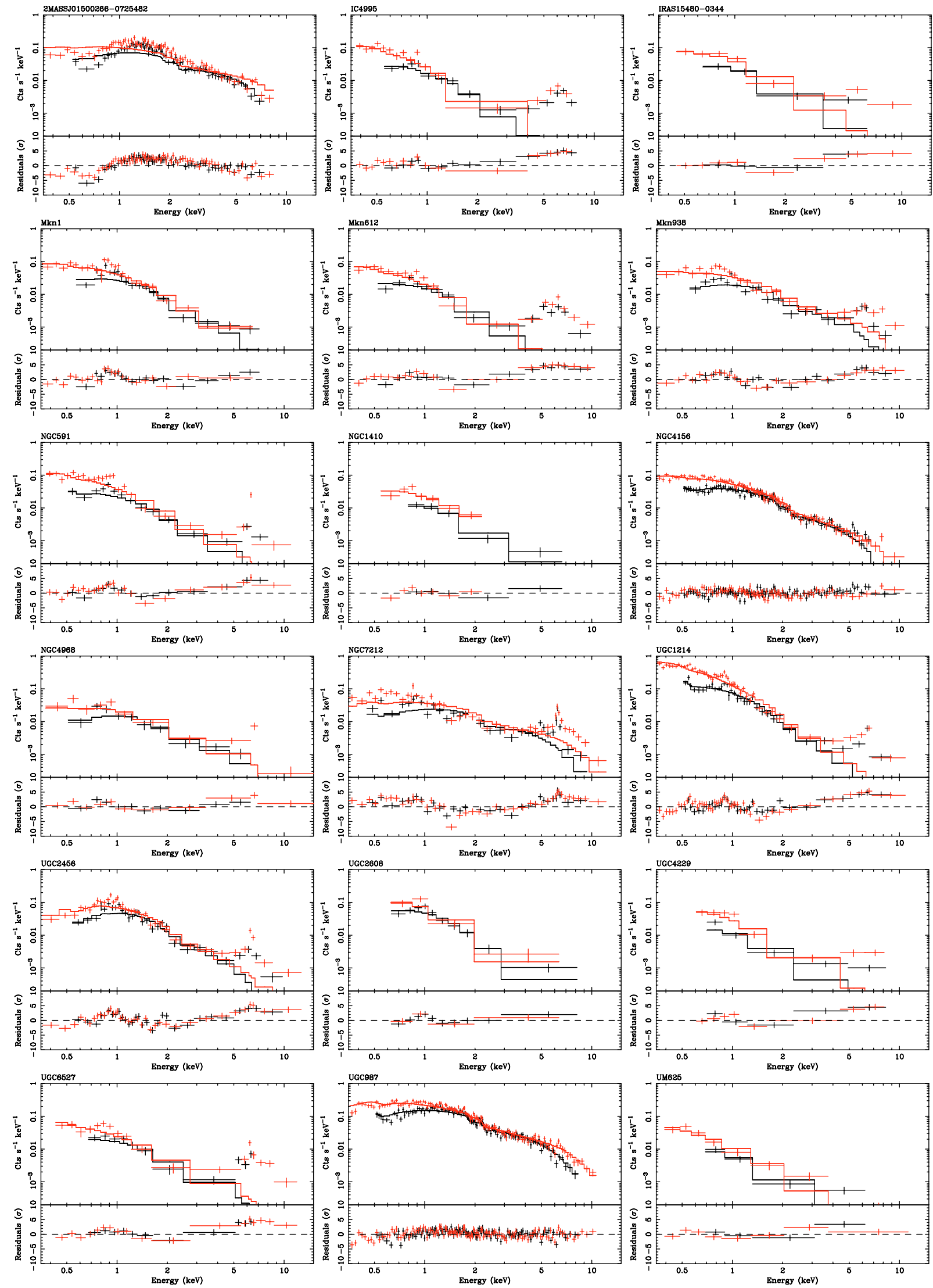

Fig. 1. Spectra (upper panels) and residuals against a power-law continuum (lower panels) for the XMM-Newton/EPIC observations of our sample. 

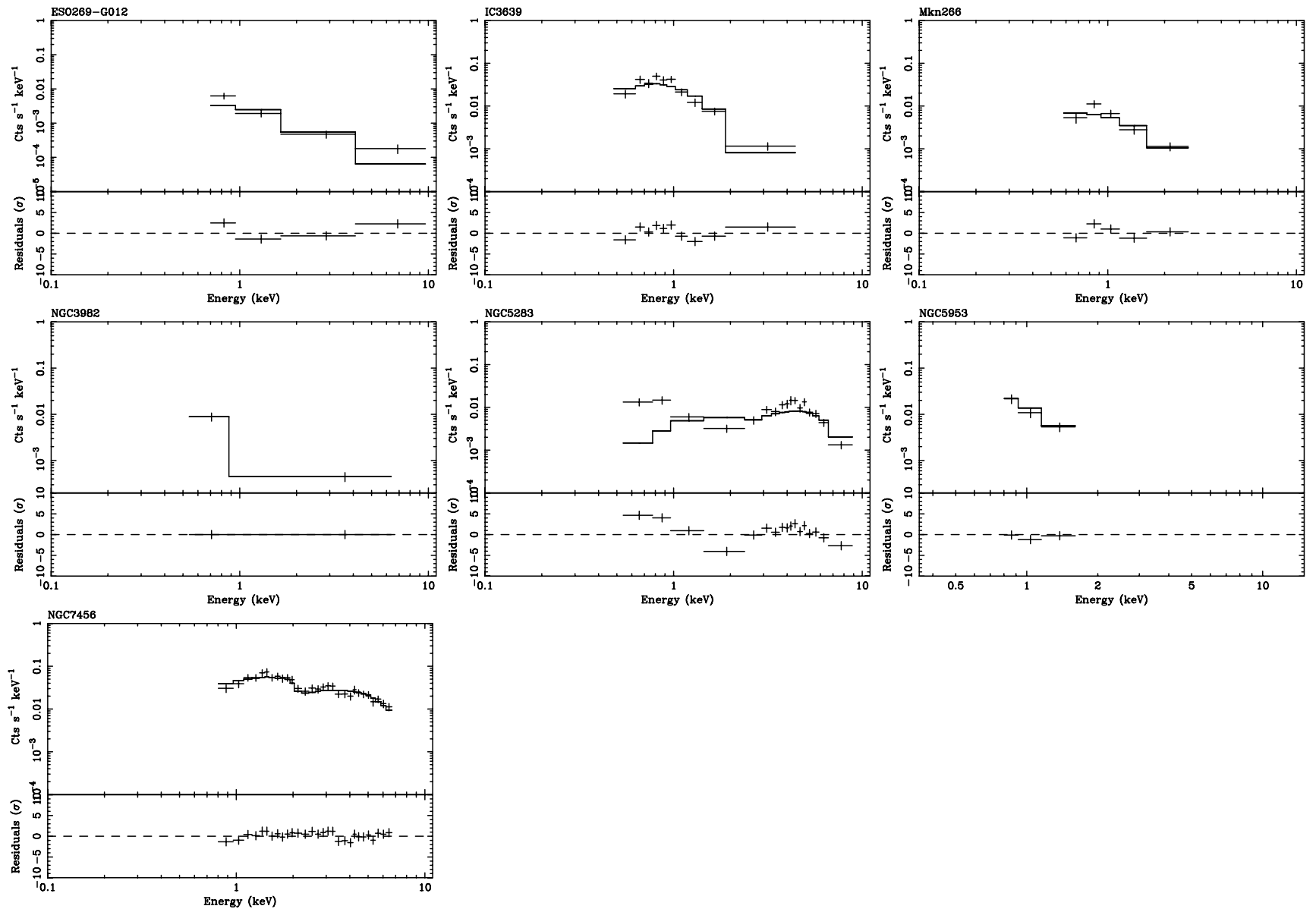

Fig. 2. Spectra (upper panels) and residuals against a power-law continuum (lower panels) for the Chandra/ACIS observations of our sample.

where $B$ is a normalization constant, and $R(E)$ is the "bare" Compton-reflection of an otherwise invisible nuclear continuum (we used the implementation pexrav in XSPEC, Magdziarz \& Zdziarski 1995). In this component, we have assumed again solar abundances, and a face-on slab. No highenergy cut-off for the intrinsic power-law continuum emission has been imposed, as it normally lies beyond the sensitive bandpass of the XMM-Newton/EPIC or Chandra/ACIS cameras (Perola et al. 2002). In principle, the Compton reflection component should have been added also to the baseline model for Compton-thin objects. However, its contribution in the pn energy bandpass is expected in these cases to be negligible, and was therefore not included for simplicity.

Observed fluxes in the $0.5-2.0 \mathrm{keV}$ and 2-10 keV energy bands, and absorption-corrected luminosity for the thermal and the AGN power-law components are shown in Table 2.

The spectra of the large majority of the sources discussed in this paper are comparatively weak, with an overall number of independent spectral channels often lower than 50. In most cases, not all the components of the baseline model are required. Moreover, in many objects it is not possible to determine whether the object is Compton-thick or -thin from a statistical point of view. In order to proper classify these objects, we need to complement the statistical results of the fits with the evaluation of two physical observables with a high diagnostic power in X-ray obscured AGN: the Equivalent Width $(E W)$ of the $\mathrm{Fe} \mathrm{K}_{\alpha}$ fluorescent line, and the ratio $T$ between the $2-10 \mathrm{keV}$ flux and the Balmer-Decrement corrected $\mathrm{O}[\mathrm{III}]$ flux.

The binning requirement imposed by the application of the $\chi^{2}$ test in the global fits may hamper the detectability of even large $E W$ emission lines in low-statistics spectra with almost no continuum besides the line. Before applying the baseline models to the broadband X-ray spectra, we have therefore fit the unbinned spectra in the $5.25-7.25 \mathrm{keV}$ energy range (where the $\mathrm{K}_{\alpha}$ fluorescent iron line complex is expected to lie for all objects in our sample), using the Cash statistics (Cash 1976; "local fits" hereinafter). Power-laws were used to independently model the continuum and the background, as the Cash method cannot be applied on spectra that do not follow Poisson statistics. Their parameters were then allowed to vary in the local fits within their confidence intervals. The properties of the iron $\mathrm{K}_{\alpha}$ lines derived from the local fits are summarized in Table $3 . \mathrm{Fe}_{\alpha}$ emission lines are detected in 6 (14) objects at a confidence level higher than $2 \sigma(1 \sigma)$. They represent the first detection of such a feature in these objects. In all cases, the centroid energies $E_{l}$ are consistent with emission from neutral or mildly ionized iron. However, in UGC 1214 an additional line with $E_{l} \simeq 6.66 \mathrm{keV}$ suggests a contribution from iron species as ionized as FE XXV. In Fig. 3 the $5.25-7.25 \mathrm{keV}$ spectra for the objects where a line is significantly detected are shown. If the $\mathrm{Fe} \mathrm{K}_{\alpha} E W$ is larger then and inconsistent with $600 \mathrm{eV}$ at the $90 \%$ confidence level 
Table 2. X-ray observed fluxes, $T$ values and absorption-corrected luminosities in the $0.5-10 \mathrm{keV}$ energy range.

\begin{tabular}{|c|c|c|c|c|c|c|}
\hline \multirow[t]{2}{*}{ Source } & \multicolumn{3}{|c|}{ Observed fluxes } & \multirow[b]{2}{*}{$T$} & \multicolumn{2}{|c|}{ Luminosities } \\
\hline & $0.5-2 \mathrm{keV}^{a}$ & $2-10 \mathrm{keV}^{a}$ & $5-10 \mathrm{keV}^{b}$ & & Thermal $^{c}$ & $\mathrm{AGN}^{d}$ \\
\hline 2MASS J01500266-0725482 & 0.30 & 0.82 & 3.7 & 1.00 & $0.9 \pm_{0.6}^{1.7}$ & $1.20 \pm_{0.07}^{0.06}$ \\
\hline ESO 209-G012 & 0.011 & 0.06 & 4.2 & $1.2^{e}$ & $1.0 \pm 0.3$ & $0.13 \pm_{0.07}^{0.10}$ \\
\hline IC 3639 & 0.10 & 0.08 & 0.39 & 0.03 & $2.4 \pm_{1.4}^{0.9}$ & $\ldots$ \\
\hline IC 4995 & 0.06 & 0.29 & 2.5 & 0.18 & $4 \pm 2$ & $\ldots$ \\
\hline IRAS 15480-0344 & 0.09 & 0.37 & 2.8 & 0.07 & $27 \pm_{15}^{233}$ & $\ldots$ \\
\hline Mkn 1 & 0.10 & 0.13 & 1.0 & 0.28 & $\ldots$ & $1.29 \pm_{1.28}^{5.50}$ \\
\hline Mkn 266 & 0.03 & $<0.007$ & $<0.03$ & $<0.03$ & $3.6 \pm_{1.0}^{1.1}$ & $\ldots$ \\
\hline Mkn 612 & 0.05 & 0.36 & 3.2 & 0.20 & $2.7 \pm_{0.5}^{0.6}$ & $7.3 \pm_{1.1}^{1.3}$ \\
\hline NGC 17 & 0.07 & 0.33 & 2.0 & 0.01 & $2.0 \pm 0.5$ & $2.16 \pm 0.06$ \\
\hline NGC 591 & 0.09 & 0.20 & 1.6 & 0.11 & $3.4 \pm_{0.9}^{1.9}$ & $\ldots$ \\
\hline NGC 1410 & 0.04 & 0.04 & 0.2 & 0.01 & $2.3 \pm_{0.9}^{1.8}$ & $0.10 \pm_{0.02}^{0.03}$ \\
\hline NGC 3982 & 0.018 & $<0.05$ & $<0.2$ & $<0.14$ & $0.057 \pm 0.0014$ & $\ldots$ \\
\hline NGC 4156 & 0.13 & 0.18 & 0.8 & $\ldots$ & $\ldots$ & $0.414 \pm 0.015$ \\
\hline NGC 4968 & 0.04 & 0.15 & 1.2 & 0.04 & $\ldots$ & $\ldots$ \\
\hline NGC 5283 & 0.04 & 1.46 & 10.3 & 2.71 & $\ldots$ & $1.7 \pm_{0.6}^{0.8}$ \\
\hline NGC 5427 & $<0.002$ & $<0.11$ & $<0.11$ & $\ldots$ & $\ldots$ & $\ldots$ \\
\hline NGC 5953 & 0.06 & $<0.006$ & $<0.02$ & $<0.01$ & $\ldots$ & $\ldots$ \\
\hline NGC 7212 & 0.09 & 0.69 & 5.6 & 0.22 & $8 \pm_{2}^{4}$ & $\ldots$ \\
\hline NGC 7465 & 0.24 & 4.15 & 29.0 & $\ldots$ & $\ldots$ & $1.0 \pm_{0.3}^{0.8}$ \\
\hline UGC 1214 & 0.32 & 0.28 & 1.7 & 0.07 & $16 \pm_{3}^{6}$ & $\ldots$ \\
\hline UGC 2456 & 0.14 & 0.36 & 2.8 & 0.07 & $\ldots$ & $\ldots$ \\
\hline UGC 2608 & 0.13 & 0.14 & 0.8 & 0.03 & $30 \pm_{11}^{9}$ & $\ldots$ \\
\hline UGC 4229 & 0.05 & 0.22 & 1.7 & 0.03 & $4.1 \pm_{1.1}^{1.2}$ & $\ldots$ \\
\hline UGC 6527 & 0.05 & 0.43 & 4.0 & 0.08 & $3.2 \pm_{1.0}^{1.1}$ & $15 \pm_{7}^{14}$ \\
\hline UGC 987 & 0.48 & 1.21 & 6.1 & 7.24 & $\ldots$ & $1.09 \pm 0.05$ \\
\hline UM 625 & 0.03 & 0.02 & 0.11 & 0.004 & $2.3 \pm_{1.0}^{2.5}$ & $\ldots$ \\
\hline
\end{tabular}

${ }^{a}$ In units of $10^{-12} \mathrm{erg} \mathrm{cm}^{-2} \mathrm{~s}^{-1}$.

${ }^{b}$ In units of $10^{-13} \mathrm{erg} \mathrm{cm}^{-2} \mathrm{~s}^{-1}$.

${ }^{c}$ In units of $10^{40} \mathrm{erg} \mathrm{s}^{-1}$.

${ }^{d}$ In units of $10^{42} \mathrm{erg} \mathrm{s}^{-1}$.

${ }^{e}$ Not corrected for Balmer decrement (measurement not available in the literature).

(the maximum observed $E W$ in "Compton-thin" objects; Turner et al. 1997; Risaliti 2002) models assuming Comptonthick obscuration were preferred, whenever statistically equivalent to those requiring a Compton-thin absorber.

Another powerful diagnostic of nuclear X-ray obscuration is the $2-10 \mathrm{keV}$ to $\mathrm{O}$ [III] flux ratio $T$. Previous studies (Maiolino et al. 1998; Bassani et al. 1999; Guainazzi et al. 2005a) have shown that objects with $T \leq 0.1$ are invariably Compton-thick, whereas objects with $T \geq 1$ are almost exclusively Compton-thin or unobscured. For all objects where the iron line $E W$ gives a constraint on the nature of the nuclear obscuration, this is consistent with that inferred from the $T$ value. This gives us confidence in using $T$ as a diagnostic of the nature of the X-ray obscuration. The values of $T$ for the objects of our sample are listed in Table 2.

A summary of the best-fit parameters and results is shown in Table 4, where the column density for "Compton-thick" objects is lower-bounded by $\sigma_{t}^{-1} \simeq 1.6 \times 10^{24} \mathrm{~cm}^{-2}$. The $E W$,
$E_{l}$ and intensity $I_{l}$ of the $\mathrm{Fe} \mathrm{K}_{\alpha}$ fluorescent lines derived from the global fits are shown in Table 3; the same properties for other lines are listed in Table 5 for the two objects (Mkn 1 and UGC 2456) where their inclusion significantly improves the quality of the fit. The meaning of the EWs corresponding to the "local" and "global" fits in Table 3 is not strictly the same. In the "local" fits, the $E W$ is calculated against the background-subtracted continuum underlying the line profile; in the "global" fits, it represents the $E W$ against the proper continuum to which it refers, i.e. the absorption-corrected primary power-law for Compton-thin, and the Compton-reflection continuum for Compton-thick objects.

\subsection{Notes on individual objects}

IC3639, NGC 1410, and UM 625: in these objects $T<0.1$, suggesting Compton-thick obscuration. The $E W$ of the $\mathrm{K}_{\alpha}$ iron line is largely unconstrained. However, the spectrum above $2 \mathrm{keV}$ 
Table 3. Fe $\mathrm{K}_{\alpha}$ properties.

\begin{tabular}{|c|c|c|c|c|c|}
\hline \multirow[b]{2}{*}{ Source } & \multicolumn{2}{|c|}{ Local fits } & \multicolumn{3}{|c|}{ Global fits } \\
\hline & $\begin{array}{c}E_{l} \\
(\mathrm{keV})\end{array}$ & $\begin{array}{c}E W \\
(\mathrm{eV})\end{array}$ & $\begin{array}{c}E_{l} \\
(\mathrm{keV})\end{array}$ & $\begin{array}{l}E W \\
(\mathrm{eV})\end{array}$ & $I_{l}^{\ddagger}$ \\
\hline 2MASS J01500266-0725482 & $6.4^{\dagger}$ & $<200$ & $6.56 \pm_{0.14}^{0.19}$ & $350 \pm_{290}^{210}$ & 0.23 \\
\hline ESO 269-G012 & $6.4^{\dagger}$ & $<700$ & $6.4^{\dagger}$ & $<3000$ & $<0.3$ \\
\hline IC 3639 & $6.39 \pm 0.07$ & $1500 \pm 1100$ & $6.4^{\dagger}$ & $<525000$ & $<174$ \\
\hline IC 4995 & $6.42 \pm_{0.05}^{0.03}$ & $1700 \pm 700$ & $6.40 \pm_{0.05}^{0.03}$ & $4300 \pm_{1100}^{1400}$ & 1.1 \\
\hline IRAS 15480-0344 & $6.41 \pm_{0.04}^{0.03}$ & $17000 \pm_{10000}^{8000}$ & $6.4^{\dagger}$ & $<2400$ & $<1.5$ \\
\hline Mkn 1 & $6.4^{\dagger}$ & $<800$ & $6.4^{\dagger}$ & $<2000$ & $<0.5$ \\
\hline Mkn 266 & $6.38 \pm_{0.06}^{0.07}$ & $6000 \pm_{4000}^{2000}$ & $6.4^{\dagger}$ & $<100000$ & $<50$ \\
\hline Mkn 612 & $6.46 \pm_{0.09}^{0.02}$ & $280 \pm_{180}^{240}$ & $6.40 \pm 0.18$ & $200 \pm \pm_{160}^{360}$ & 0.5 \\
\hline NGC 17 & $6.4^{\dagger}$ & $<1900$ & $6.4^{\dagger}$ & $<322$ & $<0.7$ \\
\hline NGC 591 & $6.41 \pm_{0.02}^{0.03}$ & $2200 \pm \pm_{600}^{700}$ & $6.47 \pm 0.03$ & $5800 \pm_{2300}^{1400}$ & 0.9 \\
\hline NGC 1410 & $6.420 \pm 0.010$ & $800 \pm \pm_{600}^{700}$ & $6.4^{\dagger}$ & $<11000$ & $<0.5$ \\
\hline NGC 3982 & $6.4^{\dagger}$ & $8000 \pm 5000$ & $6.4^{\dagger}$ & $<41000$ & $<3.0$ \\
\hline NGC 4156 & $6.4^{\dagger}$ & $<300$ & $6.4^{\dagger}$ & $<500$ & $<0.10$ \\
\hline NGC 4968 & $6.40 \pm_{0.03}^{0.02}$ & $3000 \pm_{1000}^{1200}$ & $6.41 \pm 0.02$ & $5300 \pm_{2200}^{1700}$ & 1.8 \\
\hline NGC 5283 & $6.4^{\dagger}$ & $<170$ & $6.4^{\dagger}$ & $<220$ & $<0.7$ \\
\hline NGC 5953 & $\ldots$ & $\ldots$ & $\ldots$ & $\ldots$ & $\ldots$ \\
\hline NGC 7212 & $6.41 \pm 0.03$ & $900 \pm_{300}^{200}$ & $6.41 \pm_{0.02}^{0.03}$ & $1100 \pm 200$ & 0.9 \\
\hline NGC 7465 & $6.4^{\dagger}$ & $<300$ & $6.4^{\dagger}$ & $<400$ & $<3$ \\
\hline \multirow[t]{2}{*}{ UGC 1214} & $6.41 \pm 0.05$ & $1300 \pm 500$ & $6.41 \pm 0.05$ & $2700 \pm 1000$ & 0.6 \\
\hline & $6.66 \pm 0.05$ & $700 \pm_{300}^{600}$ & $6.92 \pm 0.09$ & $15000 \pm_{8000}^{5000}$ & 0.4 \\
\hline UGC 2456 & $6.41 \pm^{0.04}$ & $1000 \pm_{400}^{300}$ & $6.42 \pm_{0.05}^{0.04}$ & $320 \pm 90$ & 0.6 \\
\hline UGC 2608 & $6.4^{\dagger}$ & $<1300$ & $6.4^{\dagger}$ & $<8000$ & $<1.2$ \\
\hline UGC 4229 & $6.4^{\dagger}$ & $<4000$ & $6.4^{\dagger}$ & $<1600$ & $<0.7$ \\
\hline UGC 6527 & $6.37 \pm_{0.05}^{0.08}$ & $350 \pm_{190}^{230}$ & $6.44 \pm 0.08$ & $100 \pm 50$ & 0.3 \\
\hline UGC 987 & $6.45 \pm 0.07$ & $180 \pm_{100}^{110}$ & $6.48 \pm_{0.07}^{0.24}$ & $120 \pm 80$ & 0.18 \\
\hline UM 625 & $6.4^{\dagger}$ & $<1100$ & $6.4^{\dagger}$ & $<6000$ & $<0.2$ \\
\hline
\end{tabular}

In units of $10^{-5} \mathrm{ph} \mathrm{cm}^{-2} \mathrm{~s}^{-1}$.

${ }^{\dagger}$ Fixed.

is typical of unobscured AGN, showing an observed spectral index $\Gamma \simeq 2$. The hard X-ray luminosity corresponding to the observed fluxes in these sources is $\simeq 5-7 \times 10^{40} \mathrm{erg} \mathrm{s}^{-1}$. Longer observations would be required to ultimately elucidate the nature of the high-energy emission in these objects. They can be reconciled with Compton-thick obscuration if scattering is dominated by a hot plasma, or substantially contaminated by serendipitous sources in the host galaxies, such as UltraLuminous X-Ray binaries or bright Supernova Remnants.

$N G C$ 7465: the application of the baseline model yields a very flat intrinsic spectral index: $\Gamma \simeq 0.7$, again unusual in AGN. A good fit is obtained if one assumes that a "standard" $(\Gamma \equiv$ 2) AGN emission is covered by a partial covering absorber, with column densities $N_{\mathrm{H}, 1} \simeq 5 \times 10^{23} \mathrm{~cm}^{-2}$, and $N_{\mathrm{H}, 2} \simeq 2 \times$ $10^{22} \mathrm{~cm}^{-2}$, with covering fractions of $15 \pm_{13}^{10} \%$ and $85 \pm_{10}^{13} \%$, respectively.

\section{Discussion}

In this section we combine the objects for which new Chandra/XMM-Newton observations have been presented in the previous section with measurements extracted from the literature on all the other objects of our sample. The sample covers a wide range of $2-10 \mathrm{keV}$ fluxes, from $3 \times 10^{-11}$ to $6 \times 10^{-15} \mathrm{erg} \mathrm{cm}^{-2} \mathrm{~s}^{-1}$ (Fig. 4).

\subsection{Correlation between $X$-ray absorption and nuclear dust content}

In Fig. 5 we show normalized robust distribution functions ${ }^{4}$ for the $E W$ of the $\mathrm{K}_{\alpha}$ iron line - used here as an indicator of X-ray

${ }^{4}$ They are histograms of the distribution functions where each measurement is represented by a Gaussian function whose mean is the measurement value and whose deviation is the measurement's statistical uncertainty. 

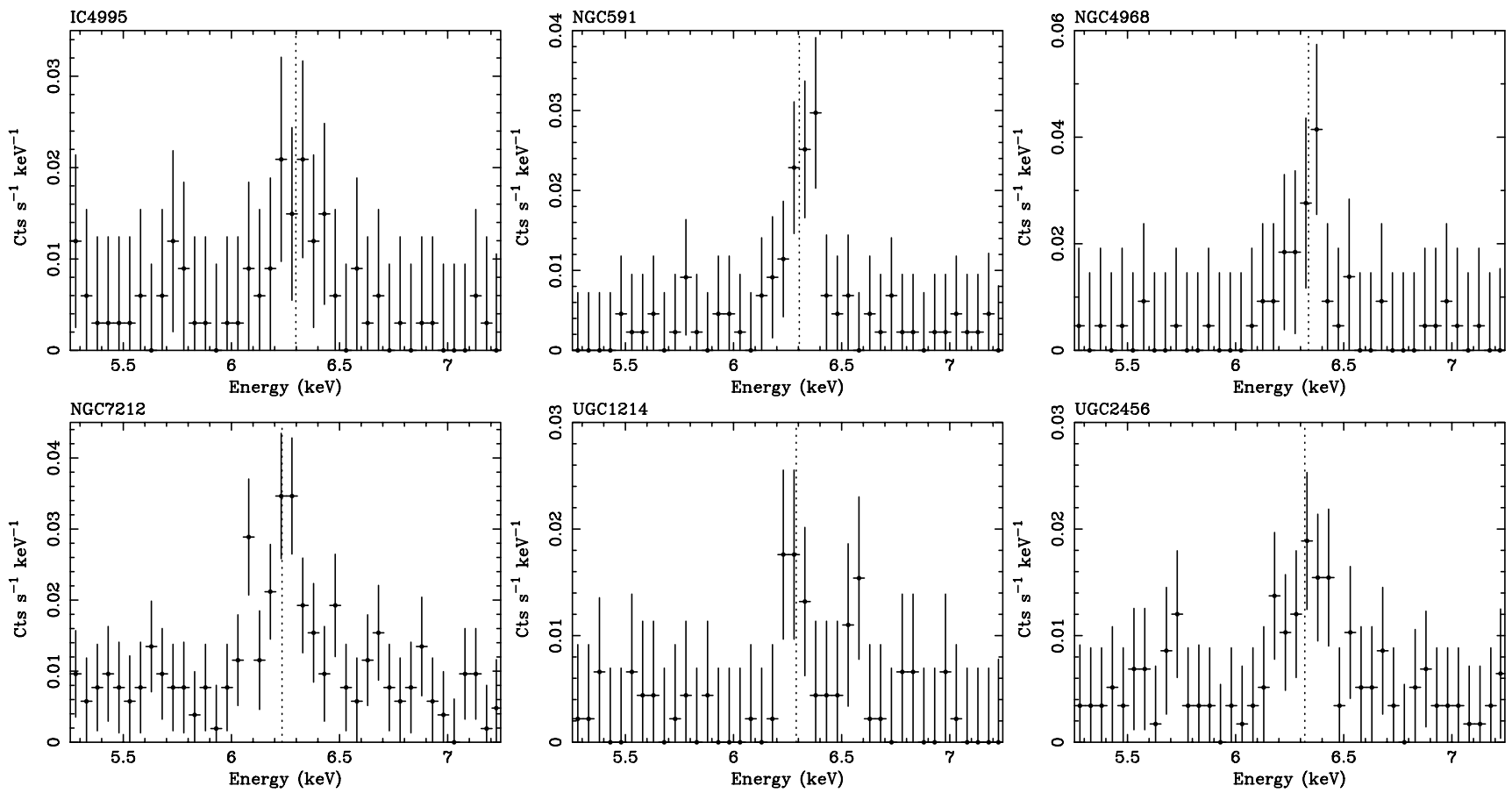

Fig. 3. Zoom of the spectra in the $5.25-7.25 \mathrm{keV}$ (observer's frame) for the sources, where the $\mathrm{K}_{\alpha}$ iron line is detected at a confidence level $\geq 2 \sigma$. The spectra are linearly binned with a constant $\delta E=50 \mathrm{eV}$. Each channel therefore corresponds approximately to one-third of a resolution element.

obscuration - for "dusty" and "non-dusty" Seyfert 2s. The probability that these distributions are drawn from the same parent population is $\sim 2 \times 10^{-8}$. The distribution weighted mean is $210 \pm 80 \mathrm{eV}$, and $1000 \pm 300 \mathrm{eV}$ for "dusty" and "non dusty" galaxies, respectively. The latter value is well above the threshold separating Compton-thin from -thick objects. This points to an average systematic difference in the X-ray absorbing column density depending on the nuclear dust content in the host galaxy.

This difference is mainly due to objects with a column density $10^{23} \leq N_{\mathrm{H}} \leq 10^{24} \mathrm{~cm}^{-2}$ ("Extreme Compton-ThiN", ECTN, objects hereafter). The ratio $R$ between ECTN with and without nuclear dust is $R_{\mathrm{ECTN}}=1.8 \pm 0.3$. For "ComptonThicK" (CTK) objects the same ratio is a factor of three lower ( $2 \sigma$ level): $R_{\mathrm{CTK}}=0.5 \pm 0.2$. The ratio calculated on the whole sample is 0.77 .

M 98 define a further group of galaxies with interesting dust absorption as those highly inclined spirals with extensive dust lanes on one side of their major axis and very little on the other side ("D-[direction]" in M 98). 7 objects in our sample fall in this class. Although they are not "nuclear dusty" according to our classification, the correlation between X-ray obscuration and the presence of dust becomes even more extreme if we include them in the "dusty" sample: $R_{\mathrm{ECTN}}=4.5 \pm 0.9, R_{\mathrm{CTK}}=$ $1.0 \pm 0.4$. The ratio calculated on the whole sample is 1.56 .

The existence of a correlation between X-ray obscuration and dust lanes in the HST images points to a relation between the host galaxy gas/dust and the matter responsible for X-ray obscuration in the innermost $\lesssim 100 \mathrm{pc}$. As already pointed out by $\mathrm{G} 01$, the correlation is probably not direct, i.e. the dust lanes seen in high-resolution HST images are not directly responsible for the X-ray obscuration, as in "DI" objects these lanes do not appear to cross the line-of-sight to the active nucleus. The connection must therefore be of an "ambient" or "evolutionary" nature, with dusty nuclear environments statistically favoring the formation of X-ray obscuring patches and filaments.

The most straightforward interpretation of these results is that Compton-thin obscuration, primarily in the column density range $10^{23} \leq N_{\mathrm{H}} \leq 10^{24} \mathrm{~cm}^{-2}$, is due to gas associated to the dust lanes. Thus, we measure a Compton-thick X-ray obscuration when our line-of-sight intercepts pc-scale compact clouds. We will still collectively call them "torii" in the following, although their detailed geometry is largely unknown. Whenever this does not occur, high-energy photons still have a high likelihood to cross gas associated with the dust lane clouds, encompassing the nuclear environment on a larger scale and with a larger covering fraction.

Alternatively, dust lanes and Compton-thick "torii" could be mutually exclusive in an AGN. In this case, one might expect that the distribution of the Mid InfraRed (MIR) luminosity differs between "dusty" and "non dusty" Seyferts. The MIR Spectral Energy Distribution of obscured AGN is dominated by "torus" reprocessing of the high-energy continuum (Alonso-Herrero et al. 2001; Efstathiou \& Siebenmorgen 2005). In Fig. 6 we show such distributions. The MIR flux density was calculated from a simple linear combination of $12 \mu$ and $25 \mu$ IRAS measurements:

$S_{\mathrm{MIR}} \equiv v_{12} S_{12}+v_{12} S_{25}$

The distributions are statistically indistinguishable. A pc-scale Compton-thick "torus" is therefore most likely present in all radio-quiet $\mathrm{AGN}$, in agreement with the ubiquity of unresolved 
Table 4. Global fit continuum spectral results. " $G_{i}$ " refers to the table number in this paper, where properties of the emission lines detected in the global fits are presented. The use of two rows for a given objects means that two thermal components are required by the fit.

\begin{tabular}{|c|c|c|c|c|c|c|}
\hline Source & $\begin{array}{c}N_{\mathrm{H}} \\
\left(10^{22} \mathrm{~cm}^{-2}\right)\end{array}$ & $\begin{array}{c}f_{\mathrm{s}} \\
(\%)\end{array}$ & $\bar{\Gamma}$ & $\begin{array}{c}k T \\
(\mathrm{keV})\end{array}$ & $\overline{G_{i}}$ & $\chi^{2} / v$ \\
\hline 2MASS J01500266-0725482 & $0.47 \pm_{0.04}^{0.05}$ & $\ldots$ & $2.09 \pm_{0.07}^{0.13}$ & $0.10 \pm 0.02$ & 3 & $118.6 / 133$ \\
\hline ESO 269-G012 & $9 \pm_{5}^{9}$ & $\ldots$ & $2^{\dagger}$ & $0.76 \pm_{0.23}^{0.16}$ & & $0.8 / 1$ \\
\hline IC 3639 & $>160$ & $\ldots$ & $3.9 \pm_{1.1}^{2.5}$ & $0.37 \pm_{0.09}^{0.30}$ & 3 & $3.3 / 4$ \\
\hline IC 3639 & & & & $1.0 \pm_{0.8}^{8.4}$ & & \\
\hline IC 4995 & $>160$ & $\ldots$ & $2.1 \pm 0.9$ & $0.096 \pm_{0.014}^{0.013}$ & 3 & $15.4 / 11$ \\
\hline IRAS 15480-0344 & $>160$ & $\ldots$ & $2^{\dagger}$ & $0.17 \pm_{0.09}^{0.07}$ & & $4.1 / 7$ \\
\hline Mkn 1 & $>110$ & $1^{\dagger}$ & $2.41 \pm_{0.11}^{0.13}$ & $\ldots$ & 5 & $36.0 / 32$ \\
\hline Mkn 266 & $>160$ & $\ldots$ & $2^{\dagger}$ & $0.78 \pm_{0.15}^{0.11}$ & & $0.5 / 1$ \\
\hline Mkn 612 & $65 \pm_{6}^{8}$ & $0.59 \pm_{0.15}^{0.26}$ & $2.40 \pm_{0.15}^{0.10}$ & $\begin{array}{c}0.089 \pm_{0.008}^{0.017} \\
0.62 \pm_{0.09}^{0.08}\end{array}$ & 3 & $32.6 / 23$ \\
\hline NGC 1410 & $>160$ & $\ldots$ & $4.3 \pm_{0.5}^{0.6}$ & $0.61 \pm_{0.16}^{0.28}$ & & $7.2 / 6$ \\
\hline NGC 17 & $40 \pm_{30}^{40}$ & $5 \pm_{3}^{2}$ & $1.85 \pm_{0.18}^{0.20}$ & $0.63 \pm_{0.11}^{0.09}$ & & $46.5 / 40$ \\
\hline NGC 591 & $>160$ & $\ldots$ & $2.4 \pm 0.9$ & $\begin{array}{l}0.09 \pm 0.04 \\
0.69 \pm 0.05\end{array}$ & 3 & $29.6 / 29$ \\
\hline NGC 3982 & $>160$ & $\ldots$ & $2^{\dagger}$ & $0.59 \pm_{0.18}^{0.13}$ & & $0.7 / 1$ \\
\hline NGC 4156 & $<0.02$ & $\ldots$ & $1.96 \pm_{0.05}^{0.08}$ & $\ldots$ & & $207.3 / 179$ \\
\hline NGC 4968 & $>160$ & $\ldots$ & $2.4 \pm_{0.4}^{0.3}$ & $\ldots$ & 3 & $23.7 / 23$ \\
\hline NGC 5283 & $15 \pm 3$ & $0.9 \pm 0.5$ & $2.3 \pm 0.5$ & $\ldots$ & & $12.5 / 13$ \\
\hline NGC 5953 & $\ldots$ & $\ldots$ & $3.9 \pm_{1.3}^{1.4}$ & $\ldots$ & & $0.6 / 1$ \\
\hline NGC 7212 & $>160$ & $\ldots$ & $1.5 \pm_{0.6}^{0.3}$ & $\begin{array}{l}0.16 \pm_{0.09}^{0.15} \\
0.72 \pm_{0.16}^{0.08}\end{array}$ & 3 & $97.0 / 65$ \\
\hline NGC 7465 & $\begin{array}{c}46 \pm_{19}^{31} \\
2.0 \pm_{0.5}^{0.6}\end{array}$ & $\begin{array}{c}1.6 \pm_{1.3}^{0.9} \\
9 \pm 3\end{array}$ & $2^{\dagger}$ & $\ldots$ & & $18.0 / 24$ \\
\hline UGC 1214 & $>160$ & $\ldots$ & $2.7 \pm 0.4$ & $\begin{array}{c}0.16 \pm 0.03^{\ddagger} \\
0.4 \pm_{0.2}^{0.4 \ddagger}\end{array}$ & 3 & $85.6 / 72$ \\
\hline UGC 2456 & $90 \pm_{30}^{60}$ & $\ldots$ & $1.97 \pm_{0.05}^{0.10}$ & $\ldots$ & 3,5 & $60.7 / 48$ \\
\hline UGC 2608 & $>160$ & $\ldots$ & $2.3 \pm_{0.3}^{1.1}$ & $\begin{array}{c}0.61 \pm_{0.46}^{0.14} \\
1.5 \pm_{0.3}^{0.8}\end{array}$ & & $8.4 / 10$ \\
\hline UGC 4229 & $>160$ & ... & $2^{\dagger}$ & $0.59 \pm_{0.21}^{0.09}$ & & $9.4 / 7$ \\
\hline UGC 6527 & $76 \pm \pm_{17}^{21}$ & $0.7 \pm_{0.6}^{0.4}$ & $2.3 \pm 0.3$ & $0.71 \pm_{0.12}^{0.14}$ & 3 & $15.3 / 22$ \\
\hline UGC 987 & $0.068 \pm 0.013$ & $\ldots$ & $1.70 \pm 0.04$ & $\ldots$ & 3 & $271.8 / 238$ \\
\hline UM 625 & $>160$ & $\ldots$ & $4.1 \pm 0.4$ & $0.23 \pm_{0.04}^{0.06}$ & & $8.1 / 6$ \\
\hline
\end{tabular}

iron fluorescent $\mathrm{K}_{\alpha}$ emission lines in their X-ray spectra (Page et al. 2003; Jiménez-Bailón et al. 2005).

The viability of dust lanes as tracer of Compton-thin X-ray obscuring gas depends on whether galactic dust outside the nucleus can produce enough extinction. Estimates from HST color maps (Mulchaey et al. 1994; Simpson et al. 1997; Ferruit et al. 2000; Quillen et al. 1999a) suggest $A_{\mathrm{V}} \simeq 0.5-1.5 \mathrm{mag}$. This corresponds to $N_{\mathrm{H}} \simeq 0.5-1.0 \times 10^{23} \mathrm{~cm}^{-2}$ for gas-todust ratios typically observed in luminous and obscured nearby AGN (Granato et al. 1997; Maiolino et al. 2001) ${ }^{5}$. The fact that

\footnotetext{
${ }^{5}$ Interestingly, the average surface density of molecular gas in the innermost $500 \mathrm{pc}$ of the Milky Way corresponds to $N_{\mathrm{H}} \simeq 4 \times 10^{22} \mathrm{~cm}^{-2}$ (Sanders et al. 1984).
}

a large fraction of "dusty" Seyfert 2 in M 98 have a rather disturbed morphology is suggestive of clumpiness of the absorbing medium, which may substantially increase the line-of-sight column density in individual objects.

Maiolino et al. (1995) suggested the presence of a "100 pc torus" in intermediate (i.e. 1.8-1.9) type Seyferts to explain the fact they they are preferentially found in edge-on hosts. Following the results of their HST survey, M98 elaborated the "Galactic Dust Model" (GDM) for obscuration - as opposed to the standard "Torus Model" - where the whole obscuration in type $2 \mathrm{AGN}$ is explained by the $100 \mathrm{pc}$ dust lanes imaged by HST. However, M98 themselves realized that this model is not applicable to Compton-thick objects, as dynamical 
Table 5. $G_{i}$ non-iron emission lines detected in the sample. The $E W$ are expressed against the scattering continuum only.

\begin{tabular}{|c|c|c|}
\hline & Mkn 1 & UGC 2456 \\
\hline \multicolumn{3}{|c|}{ FE-L, 0.7-0.8 keV } \\
\hline$E_{l}(\mathrm{keV})$ & $0.88 \pm 0.02$ & $0.805 \pm 0.016$ \\
\hline$E W(\mathrm{eV})$ & $160 \pm_{40}^{60}$ & $140 \pm 40$ \\
\hline$I_{l}^{a}$ & 2.9 & 1.1 \\
\hline \multicolumn{3}{|c|}{ NE IX, $0.915 \mathrm{keV}$} \\
\hline$E_{l}(\mathrm{keV})$ & $\ldots$ & $0.92 \pm 0.08$ \\
\hline$E W(\mathrm{eV})$ & $\ldots$ & $230 \pm_{30}^{50}$ \\
\hline$I_{l}^{a}$ & $\ldots$ & 1.4 \\
\hline \multicolumn{3}{|c|}{ NE $X, 1.02 \mathrm{keV}$} \\
\hline$E_{l}(\mathrm{keV})$ & $\ldots$ & $1.026 \pm_{0.009}^{0.014}$ \\
\hline$E W(\mathrm{eV})$ & $\ldots$ & $180 \pm 40$ \\
\hline$I_{l}^{a}$ & $\ldots$ & 0.9 \\
\hline \multicolumn{3}{|c|}{ FE-L, $1.03-1.15 \mathrm{keV}$} \\
\hline$E_{l}(\mathrm{keV})$ & $1.02 \pm 0.02$ & $1.15 \pm 0.02$ \\
\hline$E W(\mathrm{eV})$ & $130 \pm_{50}^{30}$ & $100 \pm_{40}^{30}$ \\
\hline$I_{l}^{a}$ & 0.6 & 0.6 \\
\hline \multicolumn{3}{|c|}{ MG XI, $1.34 \mathrm{keV}$} \\
\hline$E_{l}(\mathrm{keV})$ & $\ldots$ & $1.32 \pm 0.03$ \\
\hline$E W(\mathrm{eV})$ & $\ldots$ & $160 \pm_{70}^{20}$ \\
\hline$I_{l}^{a}$ & $\ldots$ & 0.46 \\
\hline \multicolumn{3}{|c|}{ SxIV, $2.00 \mathrm{keV}$} \\
\hline$E_{l}(\mathrm{keV})$ & $\ldots$ & $1.90 \pm 0.06$ \\
\hline$E W(\mathrm{eV})$ & $\ldots$ & $120 \pm_{90}^{20}$ \\
\hline$I_{l}^{a}$ & $\ldots$ & 0.18 \\
\hline
\end{tabular}

${ }^{a}$ In units of $10^{-5}$ counts erg s ${ }^{-1}$.

${ }^{b}$ Fixed.

considerations prevents the host galaxy dust lanes from providing such extreme obscuration. The GDM is therefore insufficient to explain the high fraction of local obscured AGN (Risaliti et al. 1999; this paper). This motivated Matt (2000) to propose an extension of the standard unified scenario whereby the compact, dusty "torus" intercepts our line-of-sight to Compton-thick AGN only, whereas Compton-thin obscuration could be due to gas and dust located at larger scales, possible related to the host galaxy rather than to the nuclear engine. Guainazzi et al. (2005a) and Matt et al. (2003b) have shown that some Compton-thick objects would appear to us as Compton-thin, if we observe them through a different line-ofsight. This supports the presence of a multiplicity of obscuring systems in the innermost hundreds parsecs.

The existence of extended obscuring structures on $\sim 100 \mathrm{pc}$ scale does not rule out the existence of an obscuring torus. Compact toroidal geometries (Pier \& Krolik 1993) are unable to explain the broadness of the spectrum in the $1-20 \mu \mathrm{m}$ region, and the nature of the $9.7 \mu \mathrm{m}$ silicate emission. However, several different combinations of geometry and torus physical properties are consistent with the observed IR SED of highly obscured AGN (Galliano et al. 2003), once allowance

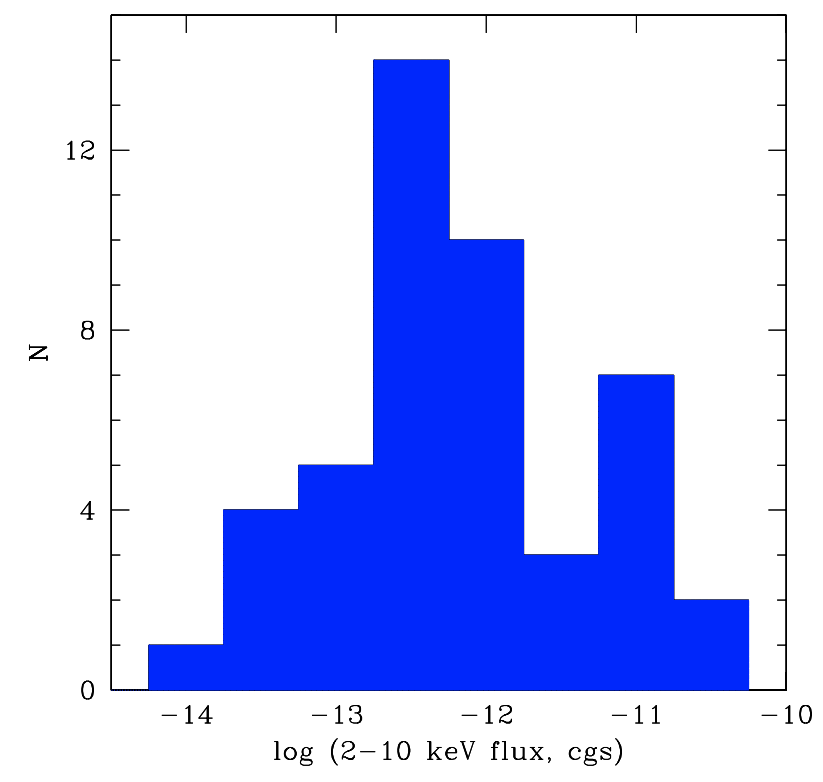

Fig. 4. 2-10 keV flux for the objects or our sample.

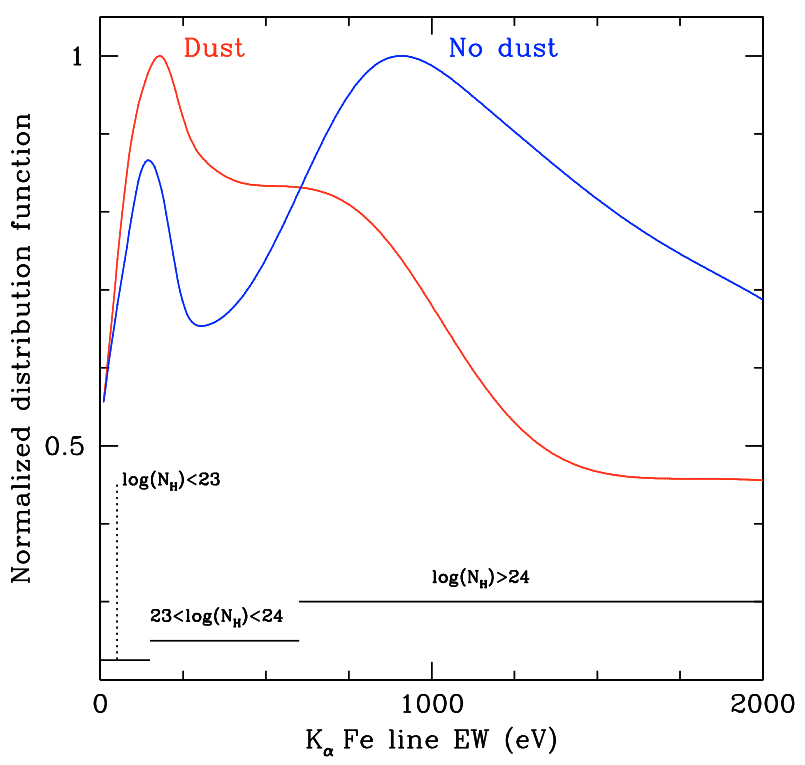

Fig. 5. Normalized distribution functions for the $\mathrm{Fe} \mathrm{K}_{\alpha}$ line $E W$ in "dusty" and "not dusty" Seyfert 2s. The solid bars indicate typical $E W$ values for Compton-thin $\left(N_{\mathrm{H}} \leq 10^{23} \mathrm{~cm}^{-2}\right)$, Extreme Comptonthin $\left(10^{23}<N_{\mathrm{H}}<10^{24} \mathrm{~cm}^{-2}\right)$, and Compton-thick $\left(N_{\mathrm{H}} \geq 10^{24} \mathrm{~cm}^{-2}\right)$ objects (cf. Fig. 9).

is made for "extended torii" (Granato \& Danese 1994), where the ratio between the outer and the inner radius is $\simeq 10-100$. Smooth (Granato et al. 1997) or clumpy (Nenkova et al. 2002) density distributions, anisotropic flares and tapered disk geometries (Efstathiou \& Rowan-Robinson 1995) are all equally successful in reproducing the data. Moreover, dust clouds might be associated as well with the extended ionizing cones. Mid-infrared emission is slightly extended in many Seyfert 2 (Maiolino \& Rieke 1995; Galliano et al. 2003). Dust lanes are sometimes associated with $\mathrm{O}[\mathrm{III}]$ ionization cones as well (Quillen et al. 1999a). Models including an optically thick torus and an optically thin "cone" of dust clouds 


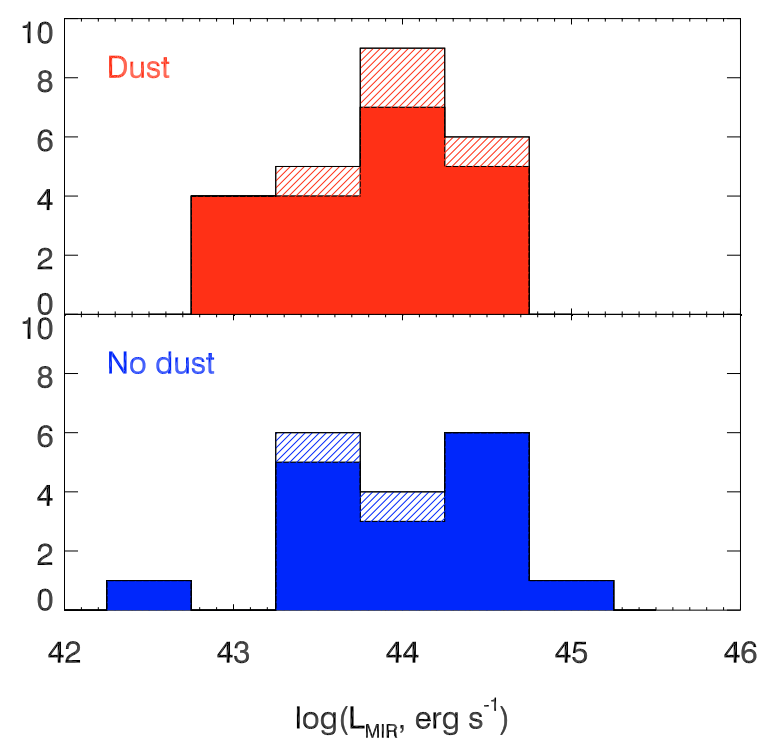

Fig. 6. MIR luminosity distribution histogram for "dusty" (upper panel) and "non-dusty" (lower panel) objects in our sample. Shaded areas indicate upper limits.

(Efstathiou \& Rowan-Robinson 1995) can as well reproduce the observed IR SEDs (Alonso-Herrero et al. 2003). Finally, radiation pressure driven starburst disks could contribute as well to the X-ray obscuration on scales $\lesssim 100 \mathrm{pc}$ (Thompson et al. 2005). All these possibilities can hardly be discriminated between even with the highest resolution imaging detectors currently available, but could be disentangled spectroscopically (Lutz et al. 2004, and references therein).

\subsection{Overall sample properties}

The parent sample, from which our sample is extracted, was selected on the basis of observational properties which should not introduce any X-ray selection effects. Our XMM-Newton program, from which most of the XMM-Newton observations discussed in this paper are drawn, carefully avoided any possible X-ray related bias. However, the inclusion of archival Chandra and XMM-Newton observations, belonging to different projects, as well as of the G01 objects may introduce a potential bias toward X-ray brighter, and therefore potentially less obscured, objects. With this caveat in mind, we review in the following Section the main X-ray spectral properties of our sample.

\subsubsection{Soft excess}

The hard X-ray continuum of type 2 Seyferts is dominated by a power-law continuum, partly suppressed by photoelectric absorption (Awaki et al. 1991; Turner et al. 1997; Risaliti 2002). In Compton-thick Seyferts, the transmitted continuum is totally suppressed below $10 \mathrm{keV}$, and the hard $\mathrm{X}$-ray continuum is accounted for by reflection of the primary nuclear emission off the inner walls of optically thick matter surrounding the nuclear environment (Matt 2000). The spectra of our sample - including those objects whose X-ray spectra are presented for the first

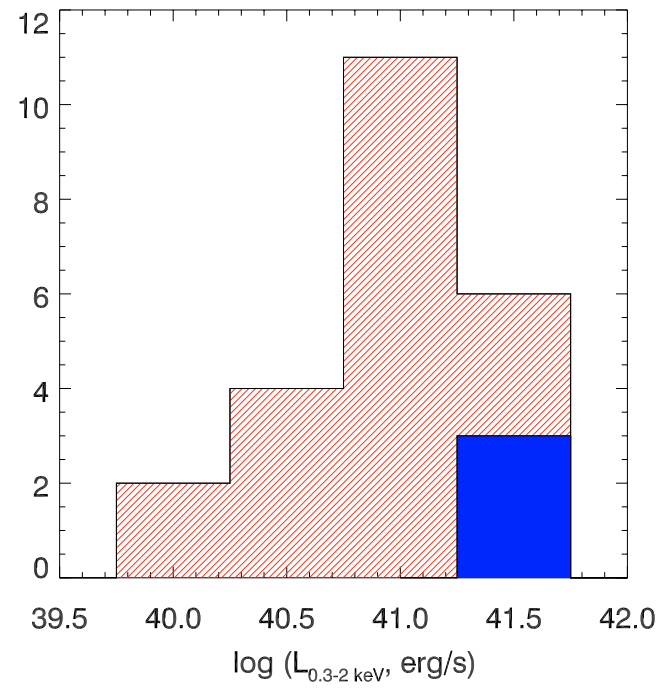

Fig. 7. Distribution function of the $0.3-2 \mathrm{keV}$ intrinsic luminosity for the sub-sample of our sample, observed by either Chandra or XMMNewton. The filled area correspond to objects, whose hard X-ray emission is covered by a column density $N_{\mathrm{H}} \leq 1.5 \times 10^{22} \mathrm{~cm}^{-2}$, implying contamination by the transmitted nuclear emission in the soft X-ray band.

time in this paper - are consistent with this scenario. Highlyobscured AGN exhibit almost invariably high soft excess emission below $2 \mathrm{keV}$ (Guainazzi et al. 2005a). In our sample, the total integrated soft $\mathrm{X}$-ray luminosity of this component ranges between $10^{40} \mathrm{erg} \mathrm{s}^{-1}$ and $4 \times 10^{41} \mathrm{erg} \mathrm{s}^{-1}$ (Fig. 7). In principle, this excess emission could be associated with unresolved sources or diffuse emission in the host galaxy, outshone by the formidable output of the AGN in unobscured objects. Although still tenable for some individual objects, this explanation cannot apply to the sample as a whole. Chandra measurements of the luminosity function in nearby spirals suggest that at most a few sources with $L_{X} \sim 10^{40} \mathrm{erg} \mathrm{s}^{-1}$ are present in each galaxy (Bauer et al. 2001; see Wolter \& Trinchieri 2004 for an intriguing exception: the Cartwheel galaxy). Likewise, there is little - if any - diffuse emission associated with spiral galaxies (at the level of a few $10^{39} \mathrm{erg} \mathrm{s}^{-1}$ in $\mathrm{M} \mathrm{31}$; Pietsch et al. 2005; Trinchieri 2004). We have searched for diffuse soft X-ray emission in the 7 objects of our sample observed with Chandra ${ }^{6}$. In 4 objects the radial profile is consistent with the instrumental PSF. In the two galaxies with the lowest $0.3-2 \mathrm{keV}$ luminosity $\left(1.5-1.7 \times 10^{40} \mathrm{erg} \mathrm{s}^{-1}\right)$ we indeed detect a large-scale lowsurface brightness component (left and central panel of Fig. 8), that in NGC 5953 extends across a significant fraction of the optical surface of the host galaxy. Finally, a peculiar object is Mkn 266 (right panel of Fig. 8), which belongs to a close interacting galaxy pair, embedded in a luminous $\left(7 \times 10^{40} \mathrm{erg} \mathrm{s}^{-1}\right)$ $\mathrm{X}$-ray halo. A detailed analysis of this intriguing system is deferred to a future paper.

In a few bright, nearby Seyfert 2 s for which high resolution spectroscopic measurements have been possible (Sako et al. 2000; Sambruna et al. 2001; Kinkhabwala et al. 2002), soft

\footnotetext{
6 The typical Point Spread Function (PSF) of the XMM-Newton observation corresponds to $\geq 25 \mathrm{kpc}$ radius.
} 

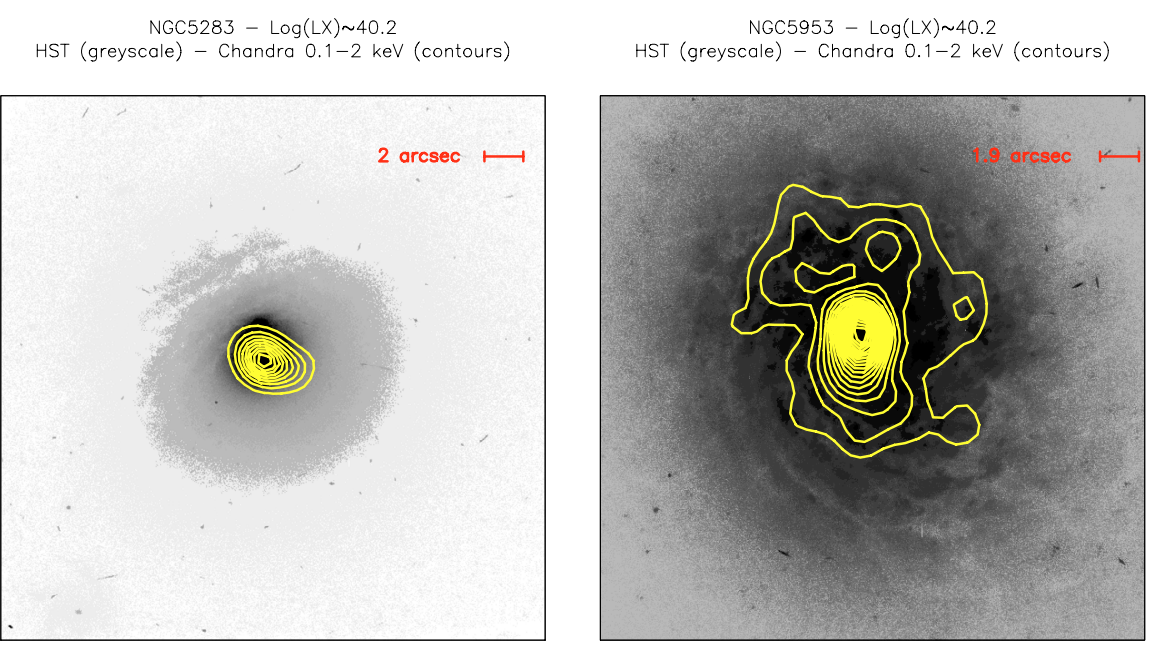

HST (greyscale) - Chandra $0.1-2 \mathrm{keV}$ (contours)
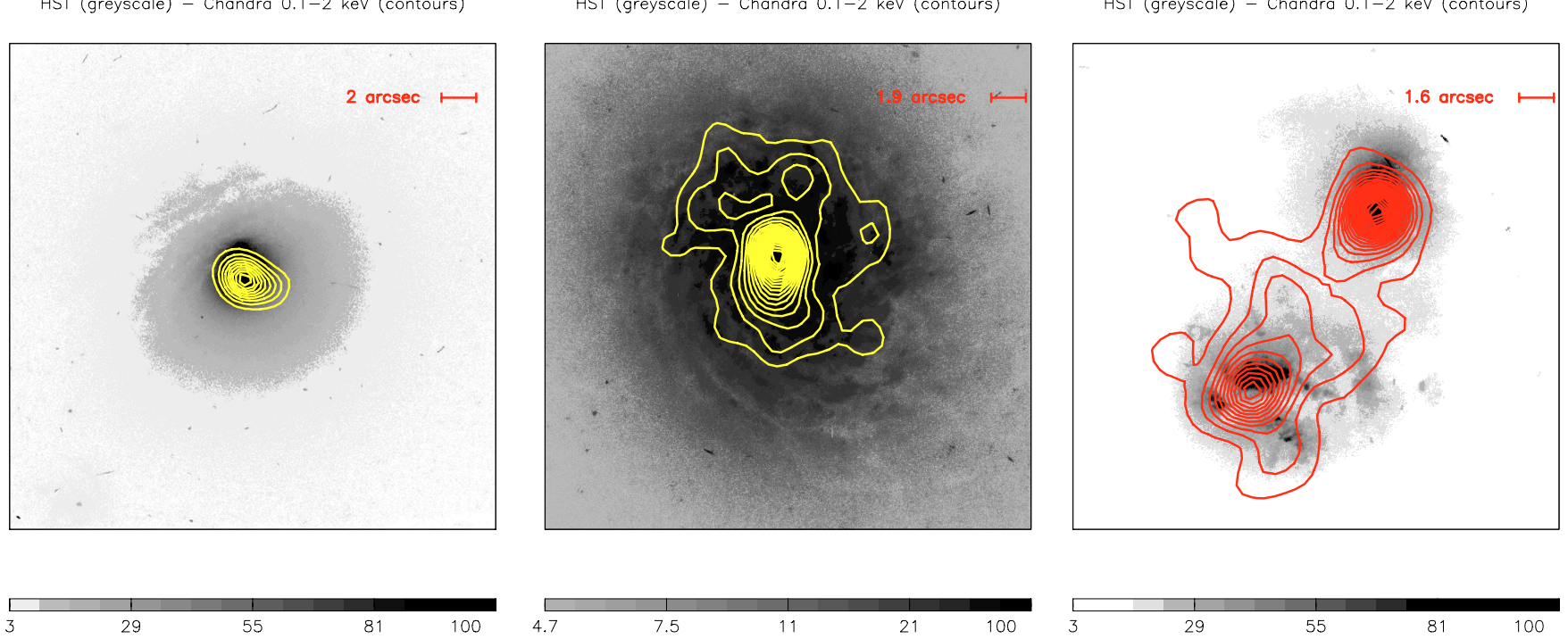

4.7

7.5

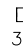

$29 \quad 55$

55

81

100

Fig. 8. HST FW606 filter (greyscale) and Chandra 0.1-2 keV images (contours) for: NGC 5283 (left), NGC 5953 (center), Mkn 266 (right). Chandra images were smoothed with a $0.75^{\prime \prime}$ Gaussian kernel. Contours levels are linearly spaced from 1 to the maximum of measured pixel counts.

$\mathrm{X}$-rays are dominated by emission lines from highly ionized species due mainly to recombination and resonant scattering following photoionization and photoexcitation. The density of the plasma is generally not high enough to substantially contribute to a scattering continuum. The striking coincidence between diffuse soft X-ray and $\mathrm{O}[\mathrm{III}]$ emission is consistent with this scenario (Bianchi et al., in preparation). However, in some low-luminosity Seyfert $2 \mathrm{~s}\left(L_{\mathrm{X}} \leq 10^{40} \mathrm{erg} \mathrm{s}^{-1}\right)$, high-resolution imaging observations with Chandra have shown that the contribution to the soft X-rays by plasma collisionally ionized in episodes of intense nuclear star formation is dominant in the soft X-ray regime (Jiménez-Bailón et al. 2003). This soft component is generally unobscured. It must therefore be produced in or by gas, extending well beyond the material absorbing the nuclear continuum. This unfortunately provides little constraint on its origin, as both nuclear starbursts and AGN-driven photoionized extended structures on scales of the order of several hundreds parsecs are common in nearby AGN (Wilson et al. 1992; Sako et al. 2000; Young et al. 2001; Bianchi et al. 2003a).

This ubiquitous soft excess may have an important impact on the classification of Compton-thin/-thick objects at cosmological redshift, if one uses standard X-ray survey colors. This point is discussed in Appendix A.

\subsection{2. $\mathrm{K}_{\alpha}$ iron lines}

It was recognized early on that fluorescent $\mathrm{K}_{\alpha}$ iron lines are a very common feature in the spectrum of highly obscured AGN (Awaki et al. 1991). In Fig. 9 we present the $E W$ of this feature (calculated against the total continuum) versus the X-ray column densities for all the objects of our sample, which do not have a lower limit on their column density, as in most cases this lower limit was inferred on the basis of the high iron line $E W-$ as discussed in Sect. 3. The two quantities are well correlated above a column density $N_{\mathrm{H}, \mathrm{tr}} \sim 10^{23} \mathrm{~cm}^{-2}$. The correlation

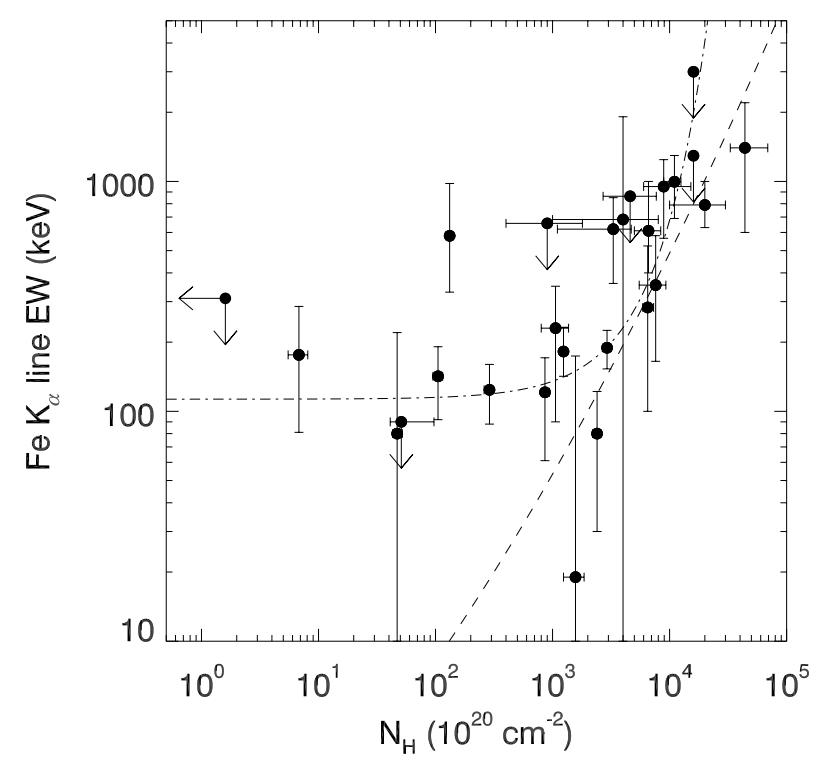

Fig. 9. X-ray obscuring column density against the iron $\mathrm{K}_{\alpha}$ fluorescent emission line $E W$ (details in text). The dashed line shows the predicted $E W$ from a uniform shell of material encompassing the continuum source (Leahy \& Creighton 1993). The dot-dashed line is the bestfit in a scenario where the line is generated by reflection off the inner wall of optically thick matter seen along an unobscured line-of-sight, while the underlying continuum is obscured by matter with a column density $N_{\mathrm{H}}$.

flattens to an almost constant $E W_{0}$ for $N_{\mathrm{H}} \leq N_{\mathrm{H}, \mathrm{rr}}$. The measured $E W \mathrm{~s}$ are significantly larger than expected in transmission from a uniform shell of material encompassing the continuum source (Leahy \& Creighton 1993). On the other hand, obscuration of continuum photons at $\simeq 6.4 \mathrm{keV}$ starts becoming significant at column densities $\simeq N_{\mathrm{H}, \mathrm{tr}}$. A censored data fit with a function:

$E W\left(N_{\mathrm{H}}\right)=E W_{0} \mathrm{e}^{\sigma_{6.4} \times N_{\mathrm{H}}}$ 


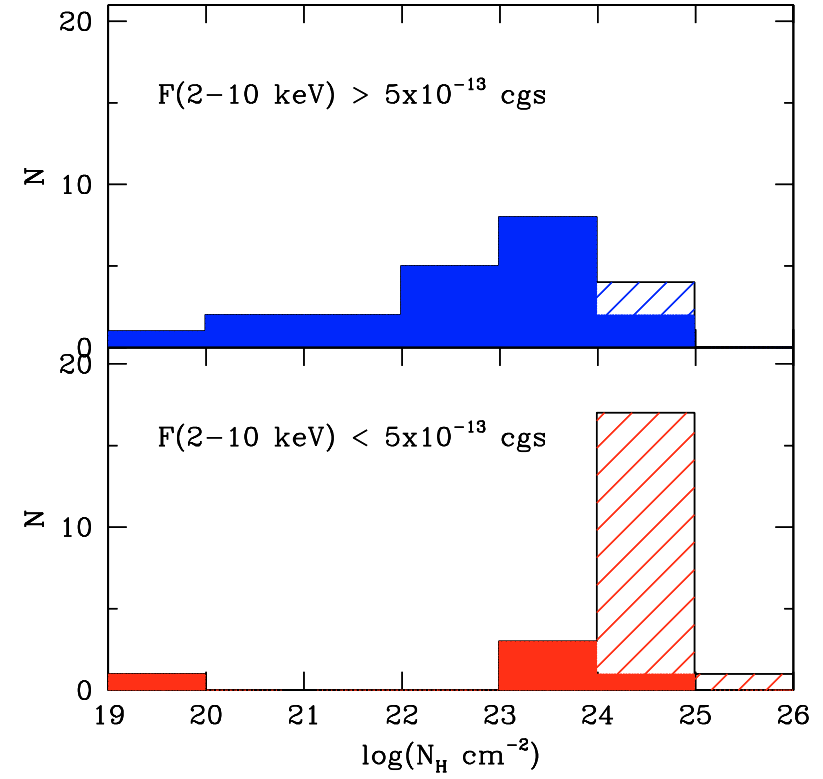

Fig. 10. $N_{\mathrm{H}}$ distribution when our sample is split according to the observed 2-10 keV flux. Shaded areas indicate lower limits.

where $\sigma_{6.4}$ is the photoelectric cross-section at $6.4 \mathrm{keV}$, yields an excellent fit: $\chi^{2} / v=17.4 / 20$. The function levels off at $E W_{0}=113 \pm 13 \mathrm{eV}$. This supports an origin of the iron line from the inner wall of the "torus" (Ghisellini et al. 1994; Krolik et al. 1994). $E W_{0}$ is in good agreement with the $E W$ of the iron $\mathrm{K}_{\alpha}$ line observed in low-luminosity $\left(L_{\mathrm{X}} \lesssim 10^{45} \mathrm{erg} \mathrm{s}^{-1}\right.$ ), X-ray unobscured quasars (Jiménez-Bailón et al. 2005). This suggests a common origin of this feature in X-ray obscured and unobscured AGN (Matt 2000).

\subsection{3. $N_{\mathrm{H}}$ distribution}

Compton-thick objects largely dominate the sample below a $2-10 \mathrm{keV}$ flux of $5 \times 10^{-13} \mathrm{erg} \mathrm{cm}^{-2} \mathrm{~s}^{-1}$. There is, however, no explicit dependency of $N_{\mathrm{H}}$ on the intrinsic AGN luminosity. If we use the $\mathrm{O}[\mathrm{III}]$ flux as a proxy for the latter quantity (it is impossible to have an estimate of the intrinsic AGN luminosity from X-ray reflection-dominated spectra), no correlation is observed with the measured column density (Fig. 11, see also Risaliti et al. 1999), in agreement with the 0th order unified models.

The total fraction of Compton-thick objects in our Seyfert 2 sample is $46 \pm 10 \%$. This is likely to represent a lower limit to the fraction on Compton-thick objects in the parent sample, due to our possible bias toward X-ray brighter, and therefore potentially less obscured, objects.

\section{Conclusions}

We have presented new low-resolution X-ray spectroscopic measurements for 26 nearby $(z<0.035)$ Seyfert 2 galaxies. We join these results with information extracted from the literature to build a sample of 49 objects. The main goal of this paper is the study of the correlation between the X-ray column

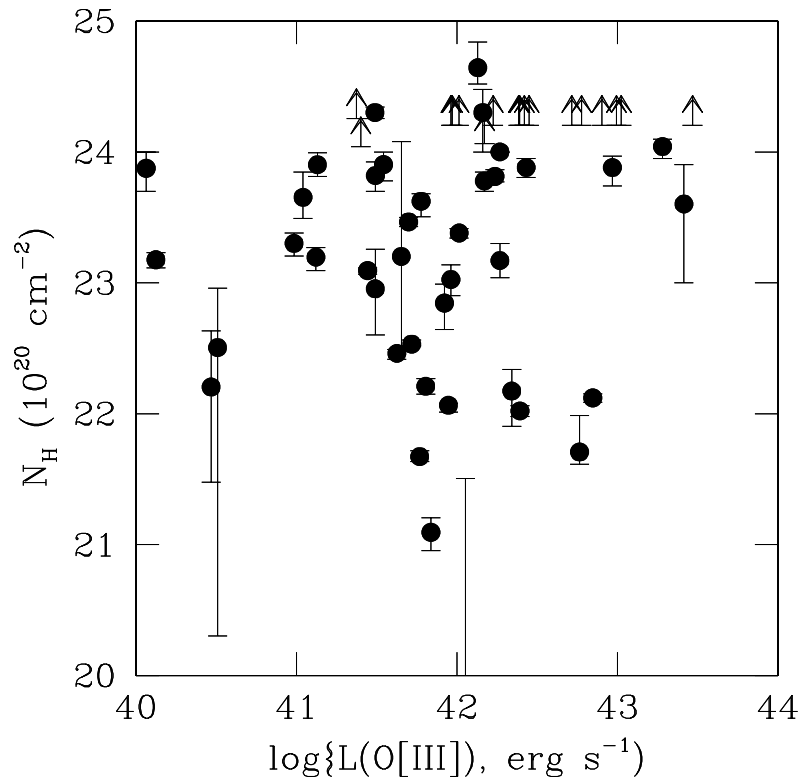

Fig. 11. O[III] luminosity versus column density for the objects of our sample.

density and the dust content on $\lesssim 100$ pc scales as imaged by the HST/WFC2. Our conclusions can be summarized as follows.

- the presence of "dust lanes" is correlated with X-ray obscuration primarily in the column density range $10^{23} \leq N_{\mathrm{H}} \leq$ $10^{24} \mathrm{~cm}^{-2}$. We interpret this result as due to the larger covering fraction of the gas associated with the dust lanes, with respect to the compact, pc-scale, Compton-thick "torus". Objects whose line-of-sight to the nucleus does not intercept the "torus" still have a non-negligible chance of being absorbed by gas on a larger scale;

- the fraction of Compton-thick objects in our sample is $46 \pm 10 \%$ and is strongly (observed-) flux dependent, as expected. No correlation exists between the amount of $\mathrm{X}$-ray obscuration and isotropic indicators of intrinsic AGN power in the luminosity range covered by our sample $\left(L_{\mathrm{X}} \lesssim\right.$ $10^{43} \mathrm{erg} \mathrm{s}^{-1}$ );

- the spectrum of highly obscured AGN is complex, with a prominent soft excess above the absorbed/reflected nuclear continuum. This excess largely dominates the overall X-ray observed energy output. This soft component is generally unobscured. This suggests an origin in gas structures, which extend beyond the material responsible for the obscuration of the nuclear continuum. The presence of this soft excess may confuse the estimate of the column density in highly-obscured, $z \lesssim 1$ objects, when standard X-ray colors are used, as shown in Appendix A.

Acknowledgements. This paper is based on observations obtained with XMM-Newton, an ESA science mission with instruments and contributions directly funded by ESA Member States and the USA (NASA). GM and GCP acknowledge financial support from MIUR under grant COFIN-03-02-23. This research has made use of data obtained through the High Energy Astrophysics Science Archive Research Center Online Service, provided by the NASA/Goddard Space Flight Center and of the NASA/IPAC Extragalactic Database (NED) which is operated by the Jet Propulsion Laboratory, California 
Institute of Technology, under contract with the National Aeronautics and Space Administration. We are grateful to M. Elitzur and N. Loiseau for stimulating discussions, and to the anonymous referee for valuable comments and suggestions.

\section{References}

Alonso-Herrero, A., Quillen, A. C., Simpson, C., Efstathiou, A., \& Ward, M. J. 2001, AJ, 131, 1369

Alonso-Herrero, A., Quillen, A. C., Rieke, G. H., Ivanov, V. D., \& Efstathiou, A. 2003, AJ, 126, 81

Anders, E., \& Grevesse, N. 1989, Geochim. Cosmochim. Acta 53, 197

Antonucci, R. 1993, ARA\&A, 31, 473

Antonucci, R. R. J., \& Miller, J. S. 1985, ApJ, 297, 621

Awaki, H., Koyama, K., Inoue, H., \& Halpern, J. O. 1991, PASJ, 43, 195

Bassani, L., Dadina, M., Maiolino, R., et al. 1999, ApJS, 121, 473

Bauer, F. E., Brandt, W. N., Sambruna, R. M., et al. 2001, AJ, 122, 182

Bianchi, S., Balestra, I., Matt, G., Guainazzi, M., \& Perola, G. C. 2003a, A\&A, 402, 141

Bianchi, S., Matt, G., Balestra, I., \& Perola, G. C. 2003b, A\&A, 407, L21

Bianchi, S., Matt, G., Balestra, I., Guainazzi, M., \& Perola, G. C. 2004, A\&A, 422, 65

Cash, W. 1976, A\&A, 52, 307

Comastri, A., Fiore, F., Vignali, C., et al. 2001, MNRAS, 327, 781

Dahari, O., \& De Robertis, M. E. 1988, ApJSS, 67, 249

de Grijp, M. H. K., Keel, W. C., Miley, G. K., Goudfrooj, P., \& Lub, P. 1992, A\&ASS, 96, 389

Efstathiou, A., \& Rowan-Robinson, M. 1995, MNRAS, 273, 649

Efstathiou, A., \& Siebenmorgen, R. 2005, A\&A, 439, 85

Elvis, M., Risaliti, G., Nicastro, F., et al. 2004, ApJ, 615, L25

Ferruit, P., Wilson, A. S., \& Mulchaey, J. 2000, 128, 139

Fiore, F., Giommi, P., Vignali, C., et al. 2001, MNRAS, 327, 771

Gabriel, C., Denby, M., Fyfe, D. J., Hoar, J., \& Ibarra, A. 2003, in Astronomical Data Analysis Software and Systems XIII, ed. F. Ochsenbein, M. Allen, \& D. Egret (San Francisco: ASP), ASP Conf. Ser., 314, 759

Galliano, E., Alloin, D., Granato, G. L., \& Villar-Martín, M. 2003, A\&A, 412, 615

Ghisellini, G., Haardt, F., \& Matt, G. 1994, MNRAS, 267, 743

Gilli, R., Salvati, M., \& Hasinger, G. 2001, A\&A, 366, 407

Granato, G. L., \& Danese, L. 1994, MNRAS, 268, 235

Granato, G. L., Danese, L., \& Franceschini, A. 1997, ApJ, 486, 147

Guainazzi, M., Fiore, F., Matt, G., \& Perola, G. C. 2001, MNRAS, 327, 323 (G01)

Guainazzi, M., Rodriguez-Pascual, P., Fabian, A. C., Iwasawa, K. \& Matt, G. 2004, MNRAS, 355, 297

Guainazzi, M., Fabian, A. C., Iwasawa, K., Matt, G., \& Fiore, F. 2005a, MNRAS, 356, 295

Guainazzi, M., Piconcelli, E., Jimenez-Bailón, E., \& Matt, G. 2005b, A\&A, 429, L9

Hasinger, G., Altieri, B., Arnaud, M., et al. 2001, A\&A, 365, L45

Kennicutt, R. C. 1992, ApJ, 388, 310

Kinkhabwala, A., Sako, M., Behar, E., et al. 2002, ApJ, 575, 732

Krolik, J. H., Madau, P., \& Życki, P. T. 1994, ApJ, 420, L57

Jiménez-Bailón, E., Santos-LLeo, M., Mas-Hesse, M., et al. 2003, ApJ, 593, 127

Jiménez-Bailón, E., Piconcelli, E., Guainazzi, M., et al. 2005, A\&A, 435,449

Lamer, G., Uttley, P., \& McHardy, I. M. 2003, MNRAS, 342, L41
Leahy, D. A., \& Creighton, J. 1993, MNRAS, 263, 314

Levenson, N. A., Weaver, K. A., Heckman, T. M., Awaki, H., \& Terashima, Y. 2005, ApJ, 618, L167

Lutz, D., Maiolino, R., Spoon, H. W. W., \& Moorwood, A. F. M. 2004, A\&A, 418, 465

Magdziarz, P., \& Zdziarski, A. A. 1995, MNRAS, 273, 837

Maiolino, R., \& Rieke, G. H. 1995, ApJ, 454, 95

Maiolino, R., Ruiz, M., Rieke, G. H., \& Keller, L. D. 1995, ApJ, 446, 561

Maiolino, R., Salvati, M., Bassani, L., Dadina, M., et al. 1998, A\&A, 338,781

Maiolino, R., Marconi, A., Salvati, M., et al. 2001, A\&A, 365, 28

Mainieri, V., Bergeron, J., Hasinger, G., et al. 2003, A\&A, 393, 425

Malkan, M. A., Gorijn, V., \& Tam, R. 1998, ApJS, 117, 25 (M 98)

Martini, P., Regan, M. W., Mulchaey, J. S., \& Pogge, R. W. 2003, ApJS, 146, 353

Matt, G. 2000, A\&A, 335, L31

Matt, G., Fabian, A. C., Guainazzi, M., et al. 2000, MNRAS, 318, 173

Matt, G., Guainazzi, M., Perola, G. C., et al. 2001, A\&A, 377, L31

Matt, G., Bianchi, S., Guainazzi, M., et al. 2003a, A\&A, 399, 519

Matt, G., Guainazzi, M., \& Maiolino, R. 2003b, MNRAS, 342, 422

Mewe, R., Gronenschild, E. H. B. M., \& van der Oord, G. H. J. 1985, A\&AS, 62, 197

Mulchaey, J. S., Koraktar, A., Ward, M. J., et al. 1994, ApJ, 436, 586

Mulchaey, J. S., Wilson, A. S., \& Tsvetanov, Z. 1996, ApJS, 102, 309

Nenvoka, M., Ivezić, Z., \& Elitzur, M. 2002, ApJ, 570, L9

Osterbrock, D. E., \& Pogge, R. W. 1987, ApJ, 323, 108

Quillen, A. C., Alonso-Herrero, A., Rieke, M. J., et al. 1999a, ApJ, 525,685

Quillen, A. C., Alonso-Herrero, A., Rieke, M. J., et al. 1999b, ApJ, 527, 696

Page, K. L., O’Brian, Reeves, J. N., \& Breeveld, A. A. 2003, MNRAS, 340, 1052

Perola, G. C., Matt, G., Cappi, M., et al. 2002, A\&A, 389, 202

Pier, E. A., \& Krolik, J. H. 1992, ApJ, 399, L23

Pier, E. A., \& Krolik, J. H. 1993, ApJ, 418, 673

Pietsch, W., Freyberg, M., \& Haberl, F. 2005, A\&A, 434, 483

Polletta, M., Bassani, L., Malaguti, G., Palumbo, G. C. C., \& Caroli, E. 1996, ApJS, 106, 399

Risaliti, G. 2002, A\&A, 386, 379

Risaliti, G., Maiolino, R., \& Salvati, M. 1999, ApJ, 522, 157

Sako, M., Kahn, S. M., Paerels, F., \& Liedahl, D. A. 2000, ApJ, 543, L115

Sambruna, R., Netzer, H., Kaspi, S., et al. 2001, ApJ, 546, L13

Sanders, D. B., Solomon, P. M., \& Scoville, N. Z. 1984, ApJ, 276, 182

Simpson, C., Wilson, A. S., Bower, G., et al. 1997, 474, 121

Strüder, L., Briel, U., Dannerl, K., et al. 2001, A\&A, 365, L18

Terlevich, R., Melnick, J., Masegosa, M., Moles, M., \& Copetti, V. F. 1991, A\&AS, 91, 285

Thompson, T. A., Quataert, E., \& Murray, N. 2005, ApJ, 630, 167

Trinchieri, G. 2004, ApSS, 289, 367

Turner, T. J., George, I. M., Nandra, K., \& Mushotzky, R. F. 1997, ApJS, 113, 23

Turner, M. J. L., Abbey, A., Arnaud, M., et al. 2001, A\&A, 365, L27

Veron-Cetty, M. P., \& Veron, P. 2003, A\&A, 2003, 412, 399

Yaqoob, T., \& Padmanabhan, U. 2004, ApJ, 608, 157

Yaqoob, T., George, I. M., Nandra, K., et al. 2001, ApJ, 546, 759

Young, A. J., Wilson, A. S., \& Shopbell, P. L. 2001, ApJ, 556, 6

Wilson, A. S., Elvis, M., Lawrence, A., \& Bland-Hawthorn, J. 1992, ApJ, 391, L75

Wolter, A., \& Trinchieri, G. 2004, A\&A, 426, 787 


\section{Online Material}




\section{Appendix A: Brief note on the use of X-ray colors to recognize Compton-thick objects}

Regardless of the detailed physical origin of the soft excess, it is interesting to estimate how such a component - which can account for a significant fraction of the observed flux in the X-ray band - may affect the identification of highly obscured AGN at $z \lesssim 1$ (at larger redshifts this component is shifted outside the energy bands where X-ray surveys are conducted). In Fig. 12 we show color plots for the sample of Compton-thick AGN discovered by the analysis described in our sample (Sect. 4) together with the optically-defined sample of Compton-thick Seyfert 2 galaxies discussed by Guainazzi et al. (2005a, the Circinus Galaxy has been excluded from the sample, as its soft X-ray emission is substantially suppressed by the Milky Way's intervening column density). For each source, we have measured the count rates in 4 different energy bands: $0.2-0.5 \mathrm{keV}$ (Ultra-Soft; US), $0.5-2.0 \mathrm{keV}$ (Soft; S), 2.0-4.5 keV (Medium, M), and 4.5-10.0 keV (Hard, $\mathrm{M})$. We have calculated the following Hardness Ratios (HRs: Hasinger et al. 2001): HR1 $\equiv(\mathrm{S}-\mathrm{US}) /(\mathrm{S}+\mathrm{US}), \mathrm{HR} 2 \equiv$ $(\mathrm{M}-\mathrm{S}) /(\mathrm{M}+\mathrm{S}), \mathrm{HR} 3 \equiv(\mathrm{H}-\mathrm{M}) /(\mathrm{H}+\mathrm{M})$. Figure 12 shows the soft color plane (HR2 versus HR1) and the hard color plane (HR3 versus HR2) for Compton-thick "local" galaxies, together with the loci occupied by "type 1" AGN, "type 2" AGN and unclassified highly obscured $\left(\log \left(N_{\mathrm{H}}\right)>10^{21.5}\right)$ sources in the XMM-Newton observation of the Lockman Hole (Mainieri et al. 2003). Compton-thick Seyfert 2 galaxies occupy a specific area of these planes, thanks to their combination of large HR3 (flat hard X-ray spectrum) and low HR2 (steep soft X-ray excess). They span a range in HR1 that encompasses typical values for both "type 1" and "type 2" AGN, suggesting a multiplicity of possible origins for the soft excess emission. In the same Figure we show which loci our "local" Comptonthick sources would occupy, if they were located at $z=1$. In the hard color plane, Compton-thick Seyfert 2 galaxies would be indistinguishable from other AGN types. A sizable fraction of them would actually occupy the same region as broad-line AGN. The soft color plane would be required to remove the degeneracy, although Compton-thick Seyfert 2s would still have a softer HR2 with respect to other less obscured AGN, which could lead to an underestimation of the column density covering their active nucleus, and consequently of the fraction of highly obscured AGN at $z \simeq 1$. 
M. Guainazzi et al.: X-ray obscured AGN in the local universe, Online Material $p 3$
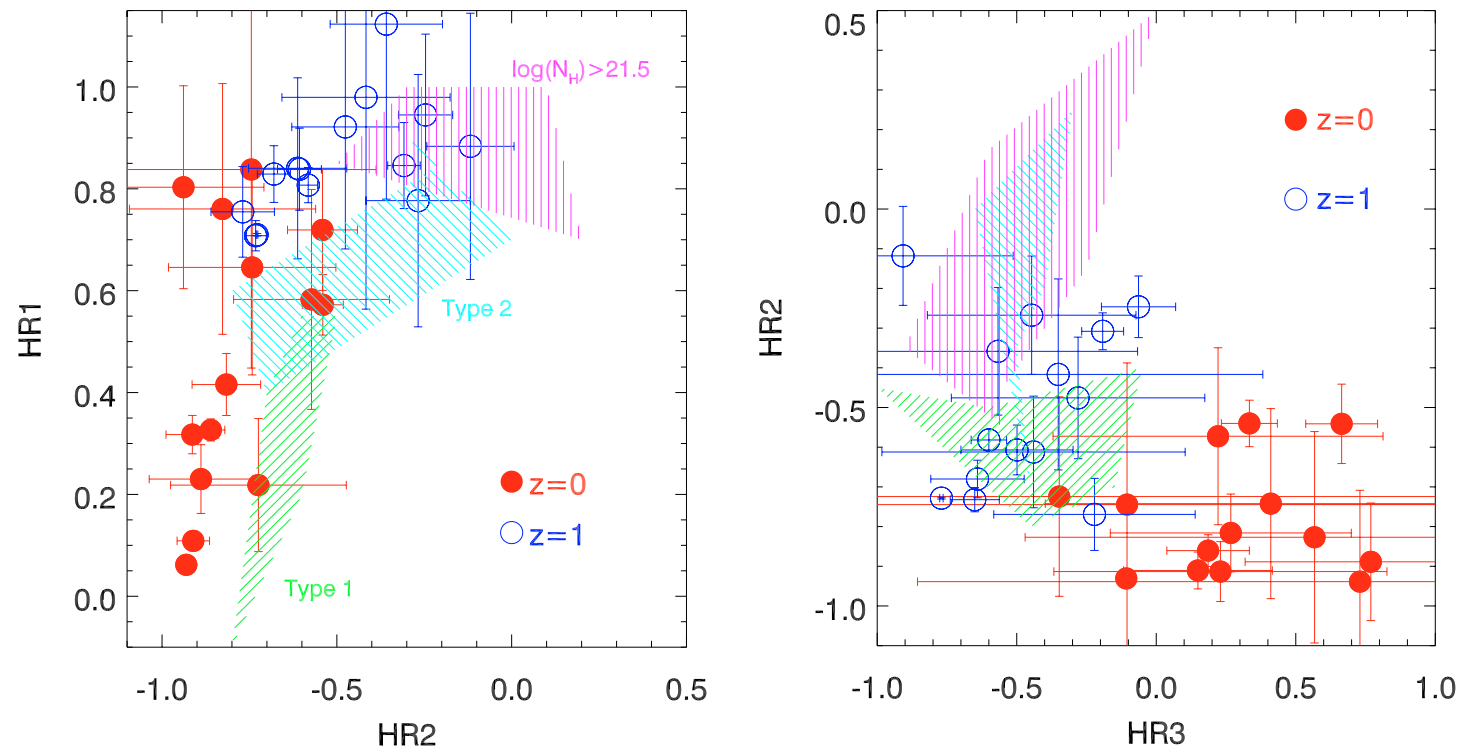

Fig. 12. Soft (left panel) and hard (right panel) color planes for the sample of Compton-thick discovered in this paper, alongside the opticallydefined Compton-thick sample of Guainazzi et al. (2005a). The Hardness Ratios (HRs) are defined in the text. The shaded areas represent the loci occupied by type 1 (left-bottom to right-top shading), type 2 (right-bottom to left-top shading), and undefined highly obscured [log $\left(N_{\mathrm{H}}\right)>$ $10^{21.5}$ ] sources (vertical shading; Mainieri et al. 2003). Filled circles correspond to Compton-thick objects at their own redshift (" $z=0$ "); empty circles to the same objects, placed at $z=1$. 